\title{
Possibility and impossibility results for selective decommitments
}

\author{
Dennis Hofheinz (CWI, Amsterdam)
}

June 11, 2008

\begin{abstract}
The selective decommitment problem can be described as follows: assume an adversary receives a number of commitments and then may request openings of, say, half of them. Do the unopened commitments remain secure? Although this question arose more than twenty years ago, no satisfactory answer could be presented so far. We answer the question in several ways:

1. If simulation-based security is desired (i.e., if we demand that the adversary's output can be simulated by a machine that does not see the unopened commitments), then security is not achievable for non-interactive or perfectly binding commitment schemes via black-box reductions to standard cryptographic assumptions. However, we show how to achieve security in this sense with interaction and a non-black-box reduction to one-way permutations.

2. If only indistinguishability of the unopened commitments from random commitments is desired, then security is not achievable for (interactive or non-interactive) perfectly binding commitment schemes, via black-box reductions to standard cryptographic assumptions. However, any statistically hiding scheme does achieve security in this sense.

Our results give an almost complete picture when and how security under selective openings can be achieved. Applications of our results include:

- Essentially, an encryption scheme must be non-committing in order to achieve provable security against an adaptive adversary.

- When implemented with our secure commitment scheme, the interactive proof for graph 3-coloring due to Goldreich et al. becomes zero-knowledge under parallel composition.

On the technical side, we develop a technique to show very general impossibility results for black-box proofs.
\end{abstract}

Keywords: cryptography, commitments, zero-knowledge, black-box separations.

\section{Introduction}

Consider an adversary $A$ that observes ciphertexts sent among parties in a multi-party cryptographic protocol. At some point, $A$ may decide, based on the information he already observed, to corrupt, say, half of the parties. By this, $A$ learns the secret keys of these parties, which allows him to open some of the observed ciphertexts. The question is: do the unopened ciphertexts remain secure? Since most encryption schemes actually constitute commitments to the respective messages, we can rephrase the question as what is known as the selective decommitment problem: assume $A$ receives a number of commitments and then may request openings of half of them. Do the unopened commitments remain secure? According to Dwork et al. [15], this question arose already more than twenty years ago in the context of Byzantine agreement, but it is still relatively poorly understood. In particular, standard cryptographic techniques (e.g., guessing which commitments are opened, or hybrid arguments) fail to show that "ordinary" commitment security against a static adversary 
guarantees security under selective openings.1 Even worse: no commitment scheme is known to be secure under selective openings.

Related work. The selective decommitment problem arises in particular in the encryption situation described above, and hence was recognized and mentioned in a number of works before (e.g., [7, 4, 8, 12, 10]). However, these works solved the problem by using (and, in fact, inventing) non-committing encryption, which circumvents the underlying commitment problem.

Dwork et al. [15] is, to the best of our knowledge, the only work that explicitly studies the selective decommitment problem. They prove that a commitment scheme which is secure under selective openings would have interesting applications. In particular, they show that the parallel composition of the graph 3-coloring protocol G3C of Goldreich et al. [20], when implemented with such a commitment scheme, satisfies a relaxed variant of zero-knowledge. They proceed to give positive results for substantially relaxed selective decommitment problems (essentially, they prove security when standard techniques can be applied, i.e., when the set of opened commitments can be guessed, or when the messages are independent). However, they leave open the question whether commitment schemes secure under (general) selective openings exist.

Our work. We answer the selective decommitment problem in several ways. First, we consider what happens if "security of the unopened commitments" means that we require the existence of a simulator $S$, such that $S$ essentially achieves what $A$ does, only without seeing the unopened commitments in the first place. We call a commitment scheme which is secure in this sense simulatable under selective openings. We show that no non-interactive or perfectly binding commitment scheme can be proved simulatable under selective openings using black-box reductions to standard assumptions. However, we also show how to construct commitment schemes which are simulatable under selective openings, under the assumption that one-way permutations exist. Our construction uses non-black-box techniques (i.e., zero-knowledge proofs) as well as interaction to circumvent our impossibility results. This solves an important open problem from Dwork et al. [15]: our schemes are the first commitment schemes provably secure under selective openings.

We proceed to consider what happens if "security" means that $A$ cannot distinguish the messages inside the unopened commitments from independent ${ }^{2}$ messages. We call a commitment scheme which is secure in this sense indistinguishable under selective openings. We show that no perfectly binding commitment scheme (interactive or not) can be proved indistinguishable under selective openings, via black-box reductions from standard assumptions. However, we also show that all statistically hiding commitment schemes are indistinguishable under selective openings.

Technically, we derive black-box impossibility results in the style of Impagliazzo and Rudich [22, but we can derive stronger claims, similar to Dodis et al. [14]. Concretely, we prove impossibility via $\forall \exists$ semi-black-box proofs from any computational assumption that can be formalized as an oracle $\mathcal{X}$ and a corresponding security property $\mathcal{P}$ which the oracle satisfies. For instance, to model one-way permutations, $\mathcal{X}$ could be a truly random permutation and $\mathcal{P}$ could be the one-way game in which a PPT adversary tries to invert a random image. We emphasize that, somewhat surprisingly, our impossibility claim holds even if $\mathcal{P}$ models security under selective openings. In that case, however, a reduction will necessarily be non-black-box, see Appendix A for a discussion.

\footnotetext{
${ }^{1}$ For instance, the probability to correctly guess an $n / 2$-sized subset of $n$ commitments is too small, and a hybrid argument would require some independence among the commitments, which we cannot assume in general.

2"independent" can of course only mean "independent, conditioned on the already opened messages"
} 
Applications. We apply our results to the adaptively secure encryption example mentioned in the beginning, and to a special class of interactive proof systems. First, we comment that an adaptively secure encryption scheme must be non-committing, or rely on non-standard techniques. Namely, whenever a committing (i.e., ciphertexts commit to messages) encryption scheme is adaptively secure, then it also is, interpreted as a (non-interactive) commitment scheme, simulatable under selective openings. Our impossibility results show that hence, a committing encryption scheme cannot be proved adaptively secure via black-box reductions from standard assumptions.

Second, we apply our results to "commit-choose-open" (CCO) style interactive proof systems such as the graph 3-coloring protocol G3C from Goldreich et al. [20. Refining the techniques of Dwork et al. [15], we prove that any CCO protocol becomes zero-knowledge under parallel composition, when implemented with a commitment scheme which is simulatable under selective openings. In particular, our (interactive, but constant-round) commitment scheme enables the parallel composability of G3C. This is surprising, given the negative results of Goldreich and Krawczyk [19] and Canetti et al. [9] for the concurrent composability limitations of (black-box) zero-knowledge proof systems. We stress that our simulator is strict polynomial-time (as opposed to expected polynomial-time simulators used, e.g., by Goldreich and Kahan [18]). We also show that a CCO protocol becomes witness-indistinguishable, even under parallel composition, when implemented with a commitment scheme which is indistinguishable under selective openings. Although somewhat less surprising, this shows the usefulness of our indistinguishability-based security definition as a reasonable fallback.

Organization. After fixing some notation in Section 2, we present in Section 3 our possibility and impossibility results for the simulation-based security definition of Dwork et al. [15]. We give an indistinguishability-based security definition, along with possibility and impossibility results in Section 4. In Section 5 and Section 6, we consider applications of our results to encryption and interactive proof systems. We discuss the role of the computational assumption in our impossibility results in Appendix A.

\section{Preliminaries}

Notation. Throughout the paper, $k \in \mathbb{N}$ denotes a security parameter. With growing $k$, attacks should be become harder, but we also allow schemes to be of complexity which is polynomial in $k$. A PPT algorithm/machine is a probabilistic algorithm/machine which runs in time polynomial in $k$. While an algorithm is stateless, a machine maintains a state across activations. A function $f=f(k)$ is called negligible if it vanishes faster than the inverse of any polynomial. That is, $f$ is negligible iff $\forall c \exists k_{0} \forall k>k_{0}:|f(k)|<k^{-c}$. If $f$ is not negligible, we call $f$ non-negligible. We say that $f$ is overwhelming iff $1-f$ is negligible. We write $[n]:=\{1, \ldots, n\}$. If $M=\left(M_{i}\right)_{i}$ is an indexed set, then we write $M_{I}:=\left(M_{i}\right)_{i \in I}$. We denote the empty (bit-)string by $\epsilon$.

\section{Commitment schemes.}

Definition 2.1 (Commitment scheme). A commitment scheme is a pair of PPT machines Com $=$ $(\mathrm{S}, \mathrm{R})$ such that the following holds:

Syntax. For any $M \in\{0,1\}^{k}, \mathrm{~S}$ (commit, $\left.M\right)$ first interacts with $\mathrm{R}$ (receive). We call this the commit phase. After that, $\mathrm{S}$ (open) interacts again with $\mathrm{R}$ (open), and $\mathrm{R}$ finally outputs a value $M^{\prime} \in\{0,1\}^{k} \cup\{\perp\}$. We call this the opening phase.

Correctness. We have $M^{\prime}=M$ always and for all $M$.

Binding. For a machine $A$, consider the following experiment $\operatorname{Exp}_{\mathrm{Com}, A}^{\text {binding }}$ : 
1. Let $A$ (commit) interact with $\mathrm{R}$ (receive),

2. let $M_{0}^{\prime}$ denote $\mathrm{R}$ 's output after interacting (on input open) with $A$ (open, 0 ),

3. rewind $A$ and $\mathrm{R}$ back to the point before step 2 ,

4. let $M_{1}^{\prime}$ denote $\mathrm{R}$ 's output after interacting (on input open) with $A$ (open, 1),

5. output 1 iff $\perp \neq M_{0}^{\prime} \neq M_{1}^{\prime} \neq \perp$.

We require that $\operatorname{Adv}_{\mathrm{Com}, A}^{\text {binding }}=\operatorname{Pr}\left[\operatorname{Exp}_{\mathrm{Com}, A}^{\text {binding }}=1\right]$ is negligible for all PPT A.

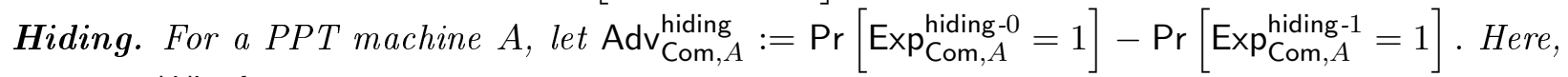

Exp Com, $A_{\text {hiding } b}$ proceeds as follows:

1. run $\left(M_{0}, M_{1}\right) \leftarrow A$ (choose) to obtain two messages $M_{0}, M_{1} \in\{0,1\}^{k}$,

2. let $\mathrm{S}$ (commit, $M_{b}$ ) interact with $A$ (receive),

3. let $b^{\prime}$ be $A$ 's final output

4. output $b^{\prime}$.

We demand that $\operatorname{Adv}_{\mathrm{Com}, A}^{\text {hiding }}$ is negligible for all PPT A.

Further, we say that Com is perfectly binding iff $\operatorname{Adv}_{\mathrm{Com}, A}^{\text {binding }}=0$ for all $A$. We say that statistically hiding iff $\operatorname{Adv}_{\mathrm{Com}, A}^{\text {hiding }}$ is negligible for all (not necessarily PPT) $A$.

Definition 2.2 (Non-interactive commitment scheme). A non-interactive commitment scheme is a commitment scheme $\mathrm{Com}=(\mathrm{S}, \mathrm{R})$ in which both commit and opening phase consist of only one message sent from $\mathrm{S}$ to $\mathrm{R}$. We can treat a non-interactive commitment scheme as a pair of algorithms rather than machines. Namely, we write $($ com, dec $) \leftarrow \mathrm{S}(M)$ shorthand for the commit message com and opening message dec sent by $\mathrm{S}$ on input $M$. We also denote by $M^{\prime} \leftarrow \mathrm{R}($ com, dec $)$ the final output of $\mathrm{R}$ upon receiving com in the commit phase and dec in the opening phase.

Note that perfectly binding implies that any commitment can only be opened to at most one value $M$. Perfectly binding (non-interactive) commitment schemes can be achieved from any oneway permutation (e.g., Blum [6]). On the other hand, statistically hiding implies that for any $M_{0}, M_{1} \in\{0,1\}^{k}$, the statistical distance between the respective views of the receiver in the commit phase is negligible. One-way functions suffice to implement statistically hiding (interactive) commitment schemes (Haitner and Reingold [21]). If we assume the existence of (families of) collisionresistant hash functions, then even constant-round statistically hiding commitment schemes exist (Damgård et al. [13], Naor and Yung [25]).

Interactive argument systems. We recall some basic definitions concerning interactive argument systems, mostly following Goldreich [17].

Definition 2.3 (Interactive proof/argument system). An interactive proof system for a language $\mathcal{L}$ with witness relation $\mathcal{R}$ is a pair of PPT machines $\mathrm{IP}=(\mathrm{P}, \mathrm{V})$ such that the following holds:

Completeness. For every family $\left(x_{k}, w_{k}\right)_{k \in \mathbb{N}}$ such that $\mathcal{R}\left(x_{k}, w_{k}\right)$ for all $k$ and $\left|x_{k}\right|$ is polynomial in $k$, we have that the probability for $\mathrm{V}\left(x_{k}\right)$ to output 1 after interacting with $\mathrm{P}\left(x_{k}, w_{k}\right)$ is at least $2 / 3$.

Soundness. For every machine $P^{*}$ and every family $\left(x_{k}, z_{k}\right)_{k \in \mathbb{N}}$ such that $\left|x_{k}\right|=k$ and $x_{k} \notin \mathcal{L}$ for all $k$, we have that the probability for $\mathrm{V}\left(x_{k}\right)$ to output 1 after interacting with $P^{*}\left(x_{k}, z_{k}\right)$ is at most $1 / 3$.

If the soundness condition holds for all PPT machines $P^{*}$ (but not necessarily for all unbounded $\left.P^{*}\right)$, then IP is an interactive argument system. We say that IP enjoys perfect completeness if $\mathrm{V}$ always outputs 1 in the completeness condition. Furthermore, IP has negligible soundness error if $\mathrm{V}$ outputs 1 only with negligible probability in the soundness condition. 
We now state what it means for an interactive proof or argument system to be zero-knowledge:

Definition 2.4 (Zero-knowledge). Let $\mathrm{IP}=(\mathrm{P}, \mathrm{V})$ be an interactive proof or argument system for language $\mathcal{L}$ with witness relation $\mathcal{R}$. IP is zero-knowledge if for every PPT machine $V^{*}$, there exists a PPT machine $S^{*}$ such that for all sequences $(x, w)=\left(x_{k}, w_{k}\right)_{k \in \mathbb{N}}$ with $\mathcal{R}\left(x_{k}, w_{k}\right)$ for all $k$ and $\left|x_{k}\right|$ polynomial in $k$, for all PPT machines $D$, and all auxiliary inputs $z^{V^{*}}=\left(z_{k}^{V^{*}}\right)_{k \in \mathbb{N}} \in\left(\{0,1\}^{*}\right)^{\mathbb{N}}$ and $z^{D}=\left(z_{k}^{D}\right)_{k \in \mathbb{N}} \in\left(\{0,1\}^{*}\right)^{\mathbb{N}}$, we have that

$$
\begin{aligned}
& \operatorname{Adv}_{V^{*}, S^{*},(x, w), D, z^{V^{*}, z^{D}}}^{\mathrm{ZK}}:=\operatorname{Pr}\left[D\left(x_{k}, z_{k}^{D},\left\langle\mathrm{P}\left(x_{k}, w_{k}\right), V^{*}\left(x_{k}, z_{k}^{V^{*}}\right)\right\rangle\right)=1\right] \\
& -\operatorname{Pr}\left[D\left(x_{k}, z_{k}^{D}, S^{*}\left(x_{k}, z_{k}^{V^{*}}\right)\right)=1\right]
\end{aligned}
$$

is negligible in $k$. Here $\left\langle\mathrm{P}\left(x_{k}, w_{k}\right), V^{*}\left(x_{k}, z_{k}^{V^{*}}\right)\right\rangle$ denotes the transcript of the interaction between the prover $\mathrm{P}$ and $V^{*}$.

Note that Definition 2.5 involves two auxiliary inputs, one input $z^{V^{*}}$ for $V^{*}$ and $S^{*}$, and one input $z^{D}$ for $D$. This deviates from the standard zero-knowledge definition (e.g., Goldreich [17, Definition 4.3.10]), in which $V^{*}, S^{*}$, and $D$ all get the same auxiliary input $z$. However, our change is without loss of generality (cf. [17, Discussion after Definition 4.3.10]). Namely, since in the standard definition, $D$ and $z$ are chosen after $V^{*}$ and $S^{*}$, and, by definition of PPT, the running time of $V^{*}$ and $S^{*}$ is polynomial in $k$ (but not in the length of $z$ ), we can pad $z$ such that only $D$ will be able to access a certain portion $z^{D}$ of $z$.

Most known interactive proof system achieve perfect completeness. Conversely, most systems do not enjoy a negligible soundness error "by nature"; their soundness has to be amplified via repetition, e.g., via sequential or concurrent composition. Thus, it is important to consider the concurrent composition of an interactive argument system:

Definition 2.5 (Concurrent zero-knowledge). Let $\mathrm{IP}=(\mathrm{P}, \mathrm{V})$ be an interactive proof or argument system for language $\mathcal{L}$ with witness relation $\mathcal{R}$. IP is zero-knowledge under concurrent composition iff for every polynomial $n=n(k)$ and PPT machine $V^{*}$, there exists a PPT machine $S^{*}$ such that for all sequences $(x, w)=\left(x_{i, k}, w_{i, k}\right)_{k \in \mathbb{N}, i \in[n]}$ with $\mathcal{R}\left(x_{i, k}, w_{i, k}\right)$ for all $i, k$ and $\left|x_{i, k}\right|$ polynomial in $k$, for all PPT machines $D$, and all auxiliary inputs $z^{V^{*}}=\left(z_{k}^{V^{*}}\right)_{k \in \mathbb{N}} \in\left(\{0,1\}^{*}\right)^{\mathbb{N}}$ and $z^{D}=\left(z_{k}^{D}\right)_{k \in \mathbb{N}} \in$ $\left(\{0,1\}^{*}\right)^{\mathbb{N}}$, we have that

$$
\begin{aligned}
& \operatorname{Adv}_{V^{*}, S^{*},(x, w), D, z^{V^{*}, z^{D}}}^{\mathrm{cZK}}:=\operatorname{Pr}\left[D\left(\left(x_{i, k}\right)_{i \in[n]}, z_{k}^{D},\left\langle\mathrm{P}\left(\left(x_{i, k}, w_{i, k}\right)_{i \in[n]}\right), V^{*}\left(\left(x_{i, k}\right)_{i \in[n]}, z_{k}^{V^{*}}\right)\right\rangle\right)=1\right] \\
& -\operatorname{Pr}\left[D\left(\left(x_{i, k}\right)_{i \in[n]}, z_{k}^{D}, S^{*}\left(\left(x_{i, k}\right)_{i \in[n]}, z_{k}^{V^{*}}\right)\right)=1\right]
\end{aligned}
$$

is negligible in $k$. Here $\left\langle\mathrm{P}\left(\left(x_{i, k}, w_{i, k}\right)_{i \in[n]}\right), V^{*}\left(\left(x_{i, k}\right)_{i \in[n]}, z_{k}^{V^{*}}\right)\right\rangle$ denotes the transcript of the interaction between $n$ copies of the prover $\mathrm{P}$ (with the respective inputs $\left(x_{i, k}, w_{i, k}\right)$ for $i=1, \ldots, n$ ) on the one hand, and $V^{*}$ on the other hand.

There exist interactive proof systems (with perfect completeness and negligible soundness error) that achieve Definition 2.5 for arbitrary NP-languages if one-way permutations exist (e.g., Richardson and Kilian [28]; see also [23, 9, 1, 16, 3] for similar results in related settings). If we assume the existence of (families of) collision-resistant hash functions, then there even exist constant-round interactive proof systems that achieve a bounded version of Definition 2.5 in which the number of concurrent instances is fixed in advance (Barak [1, Barak and Goldreich [2]).

We also recall the definition of witness indistinguishability (a relaxation of zero-knowledge) from Goldreich [17], where we chose a slightly different but equivalent formulation: 
Definition 2.6 (Witness indistinguishability). Let IP $=(\mathrm{P}, \mathrm{V})$ be an interactive proof or argument system for language $\mathcal{L}$ with witness relation $\mathcal{R}$. IP is witness indistinguishable iff for every PPT machines $V^{*}$ and $D$, all sequences $x=\left(x_{k}\right)_{k \in \mathbb{N}}, w^{0}=\left(w_{k}^{0}\right)_{k \in \mathbb{N}}$, and $w^{1}=\left(w_{k}^{1}\right)_{k \in \mathbb{N}}$ with $\mathcal{R}\left(x_{k}, w_{k}^{0}\right)$ and $\mathcal{R}\left(x_{k}, w_{k}^{1}\right)$ for all $k$ and $\left|x_{k}\right|$ polynomial in $k$, and all auxiliary inputs $z=\left(z_{k}\right)_{k \in \mathbb{N}} \in\left(\{0,1\}^{*}\right)^{\mathbb{N}}$, we have that

$$
\begin{aligned}
\operatorname{Adv}_{x, w^{0}, w^{1}, V^{*}, D, z}:=\operatorname{Pr}\left[D\left(x_{k}, z_{k},\left\langle\mathrm{P}\left(x_{k}, w_{k}^{0}\right), V^{*}\left(x_{k}, z_{k}\right)\right\rangle\right)=1\right] & \\
-\operatorname{Pr}[ & {\left[D\left(x_{k}, z_{k},\left\langle\mathrm{P}\left(x_{k}, w_{k}^{1}\right), V^{*}\left(x_{k}, z_{k}\right)\right\rangle\right)=1\right] }
\end{aligned}
$$

is negligible in $k$. Here, $\left\langle\mathrm{P}(x, w), V^{*}(x)\right\rangle$ denotes a transcript of the interaction between $\mathrm{P}$ and $V^{*}$.

Black-box reductions. Reingold et al. 27] give an excellent overview and classification of blackbox reductions. We recall some of their definitions which are important for our case. A primitive $\mathrm{P}=\left(F_{\mathrm{P}}, R_{\mathrm{P}}\right)$ is a set $F_{\mathrm{P}}$ of functions $f:\{0,1\}^{*} \rightarrow\{0,1\}^{*}$ along with a relation $R$ over pairs $(f, A)$, where $f \in F_{\mathrm{P}}$, and $A$ is a machine. We say that $f$ is an implementation of $\mathrm{P}$ iff $f \in F_{\mathrm{P}}$. Furthermore, $f$ is an efficient implementation of $\mathrm{P}$ iff $f \in F_{\mathrm{P}}$ and $f$ can be computed by a PPT machine. A machine $A$ P-breaks $f \in F_{\mathrm{P}}$ iff $R_{\mathrm{P}}(f, A)$. A primitive $\mathrm{P}$ exists if there is an efficient implementation $f \in F_{\mathrm{P}}$ such that no PPT machine $\mathrm{P}$-breaks $f$. A primitive $\mathrm{P}$ exists relative to an oracle $\mathcal{B}$ iff there exists an implementation $f \in F_{\mathrm{P}}$ which is computable by a PPT machine with access to $\mathcal{B}$, such that no PPT machine with access to $\mathcal{B}$ P-breaks $f$.

Definition 2.7 (Relativizing reduction). There exists a relativizing reduction from a primitive $\mathrm{P}=\left(F_{\mathrm{P}}, R_{\mathrm{P}}\right)$ to a primitive $\mathrm{Q}=\left(F_{\mathrm{Q}}, R_{\mathrm{Q}}\right)$ iff for every oracle $\mathcal{B}$, the following holds: if $\mathrm{Q}$ exists relative to $\mathcal{B}$, then so does $\mathrm{P}$.

Definition 2.8 ( $\forall \exists$ semi-black-box reduction). There exists a $\forall \exists$ semi-black-box reduction from $a$ primitive $\mathrm{P}=\left(F_{\mathrm{P}}, R_{\mathrm{P}}\right)$ to a primitive $\mathrm{Q}=\left(F_{\mathrm{Q}}, R_{\mathrm{Q}}\right)$ iff for every implementation $f \in F_{\mathrm{Q}}$, there exists a PPT machine $G$ such that $G^{f} \in F_{\mathrm{P}}$, and the following holds: if there exists a PPT machine $A$ such that $A^{f}$ P-breaks $G^{f}$, then there exists a PPT machine $S$ such that $S^{f}$ Q-breaks $f$.

It can be seen that if a relativizing reduction exists, then so does a $\forall \exists$ semi-black-box reduction. The converse is true when Q "allows embedding," which essentially means that additional oracles can be embedded into Q without destroying its functionality (see Reingold et al. [27, Definition 3.4 and Theorem 3.5] and Simon [29]). Below we will prove impossibility of relativizing reductions between certain primitives, which also proves impossibility of $\forall \exists$ semi-black-box reductions, since the corresponding primitives $\mathrm{Q}$ allow embedding.

\section{A simulation-based definition}

Consider the following real security game: adversary $A$ gets, say, $n$ commitments, and then may ask for openings of some of them. The security notion of Dwork et al. [15] requires that for any such $A$, there exists a simulator $S$ that can approximate $A$ 's output. More concretely, for any relation $R$, we require that $R\left(M\right.$, out $\left._{A}\right)$ holds about as often as $R\left(M\right.$, out $\left._{S}\right)$, where $M=\left(M_{i}\right)_{i \in[n]}$ are the messages in the commitments, out $A$ is $A$ 's output, and out $t_{S}$ is $S$ 's output. Formally, we get the following definition (where henceforth, $\mathcal{I}$ will denote the set of "allowed" opening sets):

Definition 3.1 (Simulatable under selective openings/SIM-SO-COM). Let $n=n(k)>0$ be polynomially bounded, and let $\mathcal{I}=\left(\mathcal{I}_{n}\right)_{n}$ be a family of sets such that each $\mathcal{I}_{n}$ is a set of subsets of $[n]$. A commitment scheme $\mathrm{Com}=(\mathrm{S}, \mathrm{R})$ is simulatable under selective openings (short SIM-SO-COM 
secure) iff for every PPT n-message distribution $\mathcal{M}$, every PPT relation $R$, and every PPT machine $A$ (the adversary), there is a PPT machine $S$ (the simulator), such that $\operatorname{Adv}_{\mathrm{Com}, \mathcal{M}, A, S, R}^{\mathrm{sim}-\mathrm{so}}$ is negligible. Here

$$
\operatorname{Adv}_{\mathrm{Com}, \mathcal{M}, A, S, R}^{\text {sim-so }}:=\operatorname{Pr}\left[\operatorname{Exp}_{\operatorname{Com}, \mathcal{M}, A, R}^{\text {sim-ro-al }}=1\right]-\operatorname{Pr}\left[\operatorname{Exp}_{\mathcal{M}, S, R}^{\text {sim-so-ideal }}=1\right],
$$

where $\operatorname{Exp}$ Com, $\mathrm{M}, A, R$ proceeds as follows:

1. sample messages $M=\left(M_{i}\right)_{i \in[n]} \leftarrow \mathcal{M}$,

2. let $A$ (receive) interact concurrently with $n$ instances $\left(\mathrm{S}_{i}\left(\text { commit, } M_{i}\right)\right)_{i \in[n]}$ of $\mathrm{S}$,

3. let $I \in \mathcal{I}$ be $A$ 's output after interacting with the $\mathrm{S}_{i}$,

4. let $A$ (open) interact concurrently with the $|I|$ instances $\left(\mathrm{S}_{i}(\mathrm{open})\right)_{i \in I}$ of $\mathrm{S}$,

5. let out $A$ denote $A$ 's final output,

6. output 1 iff $R\left(M\right.$, out $\left._{A}\right)$.

On the other hand, $\operatorname{Exp}_{\mathcal{M}, S, R}^{\text {sim-so-ideal }}$ proceeds as follows:

1. sample messages $M=\left(M_{i}\right)_{i \in[n]} \leftarrow \mathcal{M}$,

2. invoke $I \leftarrow S$ (choose) to obtain a set $I \in \mathcal{I}$,

3. invoke out ${ }_{S} \leftarrow S\left(\left(M_{i}\right)_{i \in I}\right)$,

4. output 1 iff $R\left(M\right.$, out $\left._{S}\right)$.

For simplicity, we opted not to give auxiliary input to the adversary (or to the relation $R$ ). Such an auxiliary input is a common tool in cryptographic definitions to ensure some form of composability. Not giving the adversary auxiliary input only makes our negative results stronger. We stress, however, that our positive results (Theorem 3.11 and Theorem 4.11) hold also for adversaries and relations with auxiliary input.

\subsection{Impossibility from black-box reductions}

Formalization of computational assumptions. Our first result states that SIM-SO-COM security cannot be achieved via black-box reductions from standard assumptions. We want to consider such standard assumptions in a general way that allows to make statements even in the presence of "relativizing" oracles. Thus we make the following definition, which is a special case of the definition of a primitive from Reingold et al. [27] (cf. also Section 2).

Definition 3.2 (Property of an oracle). Let $\mathcal{X}$ be an oracle. Then a property $\mathcal{P}$ of $\mathcal{X}$ is a (not necessarily PPT) machine that, after interacting with $\mathcal{X}$ and another machine $A$, finally outputs a bit $b$. For an adversary $A$ (that may interact with $\mathcal{X}$ and $\mathcal{P}$ ), we define $A$ 's advantage against $\mathcal{P}$ as

$$
\operatorname{Adv}_{\mathcal{P}, \mathcal{X}, A}^{\text {prop }}:=\operatorname{Pr}[\mathcal{P} \text { outputs } b=1 \text { after interacting with } A \text { and } \mathcal{X}]-1 / 2 .
$$

Now $\mathcal{X}$ is said to satisfy property $\mathcal{P}$ iff for all PPT adversaries $A$, we have that $\operatorname{Adv}_{\mathcal{P}, \mathcal{X}, A}^{\text {prop }}$ is negligible.

In terms of Reingold et al. [27, the corresponding primitive is $\mathrm{P}=\left(F_{\mathrm{P}}, R_{\mathrm{P}}\right)$, where $F_{\mathrm{P}}=\{\mathcal{X}\}$, and $R_{\mathrm{P}}(\mathcal{X}, A)$ iff $\operatorname{Adv}_{\mathcal{P}, \mathcal{X}, A}^{\text {prop }}$ is non-negligible. Our definition is also similar in spirit to "hard games" as used by Dodis et al. [14], but more general. We emphasize that $\mathcal{P}$ can only interact with $\mathcal{X}$ and $A$, but not with possible additional oracles. (See Appendix A for further discussion of properties of oracles, in particular their role in our proofs.) Intuitively, $\mathcal{P}$ acts as a challenger in the sense of a cryptographic security experiment. That is, $\mathcal{P}$ tests whether adversary $A$ can "break" $\mathcal{X}$ in the intended way. We give an example, where "breaking" means "breaking $\mathcal{X}$ 's one-way property". 
Example. If $\mathcal{X}$ is a random permutation of $\{0,1\}^{k}$, then the following $\mathcal{P}$ models $\mathcal{X}$ 's one-way property: $\mathcal{P}$ acts as a challenger that challenges $A$ to invert a randomly chosen $\mathcal{X}$-image. Concretely, $\mathcal{P}$ initially chooses a random $Y \in\{0,1\}^{k}$ and sends $Y$ to $A$. Upon receiving a guess $X \in\{0,1\}^{k}$ from $A, \mathcal{P}$ checks if $\mathcal{X}(X)=Y$. If yes, then $\mathcal{P}$ terminates with output $b=1$. If $\mathcal{X}(X) \neq Y$, then $\mathcal{P}$ tosses an unbiased coin $b^{\prime} \in\{0,1\}$ and terminates with output $b=b^{\prime}$.

We stress that we only gain generality by demanding that $\operatorname{Pr}[\mathcal{P}$ outputs 1 ] is close to $1 / 2$ (and not, say, negligible). In fact, this way indistinguishability-based games (such as, e.g., the indistinguishability of ciphertexts of an ideal encryption scheme $\mathcal{X}$ ) can be formalized very conveniently. On the other hand, cryptographic games like the one-way game above can be formulated in this framework as well, by letting the challenger output $b=1$ with probability $1 / 2$ when $A$ fails.

On the role of property $\mathcal{P}$. Our upcoming results state the impossibility of (black-box) security reductions, from essentially any computational assumption (i.e., property) $\mathcal{P}$. The obvious question is: what if the assumption already is an idealized commitment scheme secure under selective openings? The short answer is: "then the security proof will not be black-box." We give a detailed explanation of what is going on in Appendix A.

Stateless breaking oracles. In our impossibility results, we will describe a computational world with a number of oracles. For instance, there will be a "breaking oracle" $\mathcal{B}$, such that $\mathcal{B}$ aids in breaking the SIM-SO-COM security of any given commitment scheme, and in nothing more. To this end, $\mathcal{B}$ takes the role of the adversary in the SIM-SO-COM experiment. Namely, $\mathcal{B}$ expects to receive a number of commitments, then chooses a subset of these commitments, and then expects openings of the commitments in this subset. This is an interactive process which would usually require $\mathcal{B}$ to hold a state across invocations. However, stateful oracles are not very useful for establishing black-box separations, so we will have to give a stateless formulation of $\mathcal{B}$. Concretely, suppose that the investigated commitment scheme is non-interactive. Then $\mathcal{B}$ answers deterministically upon queries and expects each query to be prefixed with the history of that query. For instance, $\mathcal{B}$ finally expects to receive openings $d e c=\left(d e c_{i}\right)_{i \in I}$ along with the corresponding previous commitments com $=\left(\operatorname{com}_{i}\right)_{i \in[n]}$ and previously selected set $I$. If $I$ is not the set that $\mathcal{B}$ would have selected when receiving com alone, then $\mathcal{B}$ ignores the query. This way, $\mathcal{B}$ is stateless (but randomized, similarly to a random oracle). Furthermore, for non-interactive commitment schemes, this makes sure that any machine interacting with $\mathcal{B}$ can open commitments to $\mathcal{B}$ only in one way. Hence this formalization preserves the binding property of a commitment scheme, something which we will need in our proofs.

We stress, however, that this method does not necessarily work for interactive commitment schemes. Namely, any machine interacting with such a stateless $\mathcal{B}$ can essentially "rewind" $\mathcal{B}$ during an interactive commitment phase, since $\mathcal{B}$ formalizes a next-message function. Now if the commitment scheme is still binding if the receiver of the commitment can be rewound (e.g., this holds trivially for non-interactive commitment schemes, and also for perfectly binding commitment schemes), then our formalization of $\mathcal{B}$ preserves binding, and our upcoming proof works. If, however, the commitment scheme loses its binding property if the receiver can be rewound, then the following theorem cannot be applied.

We are now ready to state our result.

Theorem 3.3 (Black-box impossibility of non-interactive or perfectly binding SIM-SO-COM, most general formulation). Let $n=n(k)>0$ be arbitrary, and let $\mathcal{I}=\left(\mathcal{I}_{n}\right)_{n}$ be arbitrary such that $\mathcal{I}_{n}$ is a set of subsets of $[n]$ and $\left|\mathcal{I}_{n}\right|$ is super-polynomial in $k .^{3}$ Let $\mathcal{X}$ be an oracle that satisfies property

\footnotetext{
${ }^{3}$ e.g., one could think of $n=2 k$ and $\mathcal{I}_{n}=\{I \subseteq[n]|| I \mid=n / 2\}$ here
} 
$\mathcal{P}$. Then there is a set of oracles relative to which $\mathcal{X}$ still satisfies property $\mathcal{P}$, but there exists no non-interactive or perfectly binding commitment scheme which is simulatable under selective openings.

Proof. First, let $\mathcal{R O}$ be a random oracle (i.e., a random function $\{0,1\}^{*} \rightarrow\{0,1\}^{k}$ ). When writing $\mathcal{R O}\left(x_{1}, \ldots, x_{\ell}\right)$, we assume that $\mathcal{R O}$ 's input $x_{1}, \ldots, x_{\ell}$ is encoded in a prefix-free way, such that all individual $x_{i}$ can be efficiently reconstructed from $\mathcal{R O}$ 's input. Furthermore, to derive our second oracle $\mathcal{B}$, first consider the following machine $B$ :

1. Upon receiving Com as input, interpret Com as the description of two machines $(S, R)$ as in Definition 2.1. Then, concurrently receive $n$ Com-commitments, indexed by $i \in[n]$.

2. When all commitments are received, output a uniformly chosen $I \in \mathcal{I}$.

3. Engage in $|I|$ concurrent opening phases for the Com-instances with $i \in I$. If all openings are valid (i.e., every receiver instance with $i \in I$ outputs some $M_{i} \neq \perp$ ), return the set of all $X \in\{0,1\}^{k / 3}$ such that $M_{i}=\mathcal{R O}($ Com, $i, X)$ for all $i \in I$.

Unfortunately, we cannot use $B$ directly in our proof, since $B$ is stateful, and black-box separations require stateless oracles. So let $\mathcal{B}$ be the oracle that evaluates $B$ 's next-message function. Formally, $\mathcal{B}$ expects queries of the form $h=\left(h_{i}\right)_{i \in[\ell]}$. Upon each such query, $\mathcal{B}$ invokes a fresh copy of $B$, and feeds it input messages $h_{1}$ up to $h_{\ell}$ successively, ignoring the respective answers of $B$. Finally, $\mathcal{B}$ outputs $B$ 's answer to the last input $h_{\ell}$. The random coins used for $B$ in a given activation are supplied by $\mathcal{B}$ as a random (but deterministic) function of the previous message history of $B$. This way, $\mathcal{B}$ itself is randomized but stateless, and can be used to emulate interactions with $B$. (In fact, $\mathcal{B}$ models a $B$ which can be rewound.)

We now comment on the description of Com that $\mathcal{B}$ receives. Com describes two machines $\mathrm{S}$ and $\mathrm{R}$, which may make arbitrary oracle calls (even recursive $\mathcal{B}$-queries). We make no requirement that Com describes a hiding, binding, or correct commitment scheme. However, we do require that $\mathrm{S}$ and $\mathrm{R}$ are PPT whenever the description Com is generated by a PPT algorithm. We achieve this with a suitable padding: We require all $\mathcal{B}$-queries $h$ are prefixed with $1^{\ell}$, where $\ell$ bounds $\mathcal{B}$ 's running time on input $h$. Here, we count any oracle query with input $x$ as $|x|$ computational steps, and the final computation of all $X$ as one step. This way, not even recursive $\mathcal{B}$-queries consume more than overall $\ell$ steps (not measuring the time needed to parse $\ell$ ), while any PPT commitment scheme Com can still be encoded efficiently.

For a query $h=\left(h_{i}\right)_{i \in[\ell]}$, let $I^{h} \in \mathcal{I}$ and $M_{I^{h}}^{h}=\left(M_{i}^{h}\right)_{i \in I^{h}}$ denote the variables from the corresponding interaction with $B$. For a commitment scheme Com and a machine $A$, we say that $A$ breaks $\mathrm{Com}^{*}$ in $\mathcal{B}$ iff $A$ manages to output two queries $h=\left(h_{i}\right)_{i \in[\ell]}$ and $h^{\prime}=\left(h_{i}^{\prime}\right)_{i \in\left[\ell^{\prime}\right]}$ such that the following holds.

- $h_{i}=h_{i}^{\prime}$ for all $i \leq i^{I}$, where $i^{I}$ is the (unique) index for which $\mathcal{B}\left(\left(h_{i}\right)_{i \in\left[i^{I}\right]}\right)$ outputs $I^{h} \in \mathcal{I}$.

- There is an index $j \in[n]$ such that $\perp \neq M_{j}^{h} \neq M_{j}^{h^{\prime}} \neq \perp$.

In other words, this holds if $A$ manages to produce interactions with $B$ in which the same commitment is opened in different ways.

From here on, fix a (hiding and binding) commitment scheme Com* $=\left(\mathrm{S}^{*}, \mathrm{R}^{*}\right)$, such that Com* is non-interactive or perfectly binding (or both). We first show that our modeling of $\mathcal{B}$ preserves the binding property of Com*.

Lemma 3.4. No PPT adversary $A$ breaks $\mathrm{Com}^{*}$ in $\mathcal{B}$ with non-negligible probability.

Proof. If Com* is perfectly binding, there never exists a commitment for which two different openings are possible (as long as the receiver acts honestly). Hence there simply are no $h$ and $h^{\prime}$ as required to break the binding property of $\mathrm{Com}^{*}$ in $\mathcal{B}$. On the other hand, if $\mathrm{Com}^{*}$ is non-interactive, then $A$ must find a non-interactive commitment com along with two non-interactive openings $\operatorname{dec}_{1}$ and 
$\operatorname{dec}_{2}$ in order to break $\mathrm{Com}^{*}$ in $\mathcal{B}$. The (ordinary) binding property of $\mathrm{Com}^{*}$ implies that this is not efficiently possible.

Now consider the $n$-message distribution $\mathcal{M}^{*}=\left\{\left(\mathcal{R O}\left(\mathrm{Com}^{*}, i, X^{*}\right)\right)_{i \in[n]}\right\}_{X^{*} \in\{0,1\}^{k / 3}}$ (i.e., $\mathcal{M}^{*}$ chooses $X^{*} \in\{0,1\}^{k / 3}$ uniformly and then sets $M_{i}^{*}=\mathcal{R O}\left(\mathrm{Com}^{*}, i, X^{*}\right)$ for all $\left.i\right)$.

Lemma 3.5. There is an adversary $A$ that outputs out ${ }_{A}=M^{*}$ with overwhelming probability in the real SIM-SO-COM experiment $\operatorname{Exp}_{\mathrm{Com}}^{\text {sim-so-real }},{ }_{A, R}$. Here $M^{*}$ denotes the full message vector sampled from $\mathcal{M}^{*}$ by the experiment.

Proof. Let $A$ be the SIM-SO-COM adversary on Com* that relays between its interface to the SIMSO-COM experiment and $\mathcal{B}$ as follows. We silently assume that $A$ prefixes queries to $\mathcal{B}$ with the respective message history, and applies a padding as described above.

1. Initially, send Com* to $\mathcal{B}$.

2. Relay the $n$ commitments from the SIM-SO-COM experiment to $\mathcal{B}$.

3. Upon receiving $I^{*} \in \mathcal{I}$ from $\mathcal{B}$, send $I^{*}$ to the SIM-SO-COM experiment.

4. Upon receiving $\left|I^{*}\right|$ openings from the experiment, relay these openings to $\mathcal{B}$.

5. Finally, upon receiving a singleton set $\left\{X^{*}\right\}$ from $\mathcal{B}$, return out ou $=\left(\mathcal{R O}\left(\mathrm{Com}^{*}, i, X^{*}\right)\right)_{i \in[n]}$.

If $\mathcal{B}$ returns a set of larger size, return out A $_{A}=\perp$.

By construction of $\mathcal{M}^{*}$ and $\mathcal{B}$, it is clear that out $A=M^{*}$ unless $\mathcal{B}$ returns multiple $X$ (which happens only with negligible probability by a counting argument).

Lemma 3.6. Any given PPT simulator $S$ will output out ${ }_{S}=M^{*}$ in the ideal SIM-SO-COM experiment $\operatorname{Exp}_{\mathcal{M}, S, R}^{\text {sim-so-ideal }}$ only with negligible probability.

Proof. Fix a PPT $S$. We claim that in the ideal SIM-SO-COM experiment, $S$ has a view that is almost statistically independent of $X^{*}$, and hence will output out ${ }_{S}=M^{*}$ only with negligible probability. To show the claim, denote by $I^{*}$ the subset that $S$ submits to the SIM-SO-COM experiment, and by $M_{I^{*}}^{*}$ the messages that $S$ receives back. Denote by $\operatorname{Com}^{j}, I^{j}, M_{I^{j}}^{j}$ the corresponding values used in $S$ 's $j$-th query $h^{j}=\left(h_{i}^{j}\right)_{i \in\left[\ell^{j}\right]}$ to $\mathcal{B}$. We first define and bound a number of "bad" events:

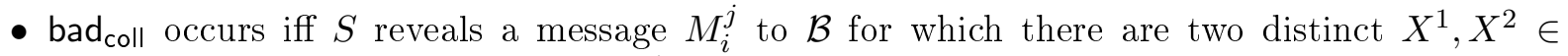
$\{0,1\}^{k / 3}$ with $\mathcal{R O}\left(\mathrm{Com}^{j}, i, X^{1}\right)=M_{i}^{j}=\mathcal{R O}\left(\mathrm{Com}^{j}, i, X^{2}\right)$.

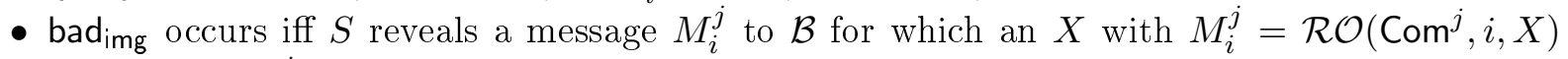
exists, but $M_{i}^{j}$ has not been obtained through an explicit $\mathcal{R O}$-query (by either $S$ or the SIM-SO-COM experiment).

- bad $_{\text {bind }}$ occurs iff $\left(\mathrm{Com}^{j}, I^{j}, M_{I^{j}}^{j}\right)=\left(\mathrm{Com}^{*}, I^{*}, M_{I^{*}}^{*}\right)$ for some $j$.

- $\operatorname{bad}:=\operatorname{bad}_{\text {coll }} \vee$ badimg $_{\text {in }} \vee$ bad $_{\text {bind }}$.

These events occur only with negligible probability: informally, bad coll implies a collision among $2^{k / 3}$ uniformly distributed $k$-bit values, which is ruled out by a birthday bound. badimg means that $S$ guessed an element of a very sparse set. Finally, bad bind means that $S$ broke Com*'s binding property (or, rather, $S$ broke $\mathrm{Com}^{*}$ in $\mathcal{B}$ ). A detailed proof can be found in Lemma 3.7 below.

Now consider the following machine $B^{\prime}$ which is almost identical to $B$ (the difference to $B$ is emphasized):

1. Upon receiving Com as input, interpret Com as the description of two machines $(S, R)$ as in Definition 2.1. Then, concurrently receive $n$ Com-commitments, indexed by $i \in[n]$.

2. When all commitments are received, output a uniformly chosen $I \in \mathcal{I}$. 
3. Engage in $|I|$ concurrent opening phases for the Com-instances with $i \in I$. If all openings are valid (i.e., every receiver instance with $i \in I$ outputs some $M_{i} \neq \perp$ ), proceed as follows. If every $M_{i}$ is the result of an $\mathcal{R O}(\mathrm{Com}, i, X)$-query of $S$ (for the same $X \in\{0,1\}^{k / 3}$ ), then output $\{X\}$. Otherwise, output $\emptyset$.

Denote by $\mathcal{B}^{\prime}$ the oracle that evaluates $B^{\prime}$ 's next-message function. We first remark that $\mathcal{B}^{\prime}$ can be efficiently simulated inside $S: \mathcal{B}^{\prime}$ running time is (roughly) the same as $\mathcal{B}^{\prime}$ s running time, if we count oracle queries and the final computation of the $X$ as above. Furthermore, by definition, the output of $\mathcal{B}$ and $\mathcal{B}^{\prime}$ can differ only if

- there are multiple $X$ with $M_{i}=\mathcal{R O}($ Com, $i, X)$ for some $i \in I$, or

- for some $i \in I, M_{i}$ is not the result of an explicit $\mathcal{R O}$-query of $S$, but there exists an $X$ with $M_{i}=\mathcal{R O}($ Com, $i, X)$ for all $i \in I$.

Suppose bad does not occur. Then $\neg$ bad $_{\text {coll }}$ ensures that no multiple $X$ with $M_{i}=\mathcal{R O}(\mathrm{Com}, i, X)$

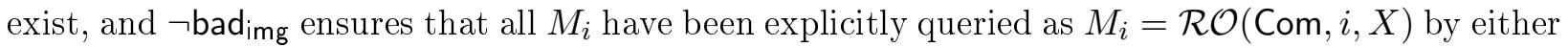
$S$ or the SIM-SO-COM experiment. Now since the SIM-SO-COM experiment makes only queries of the form $M_{i}^{*}=\mathcal{R O}\left(\mathrm{Com}^{*}, i, X^{*}\right)$, this means that $\mathcal{B}$ and $\mathcal{B}^{\prime}$ can only differ if $\mathrm{Com}=\mathrm{Com}^{*}$, and if $M_{I}$ contains some $M_{i}$ from $M_{I^{*}}^{*}$. On the other hand, $\neg$ bad $_{\text {bind }}$ implies that then, $M_{I}$ must also contain some $M_{i^{\prime}}$ not contained in $M_{I^{*}}^{*}$. By $\neg$ badimg $_{\text {img }}$, then $M_{i^{\prime}}$ must have been explicitly queried by $S$ through $M_{i^{\prime}}=\mathcal{R O}\left(\mathrm{Com}^{*}, i^{\prime}, X^{*}\right)$, for the same $X^{*}$ as chosen by the SIM-SO-COM experiment to generate $M_{i}^{*}=\mathcal{R O}\left(\mathrm{Com}^{*}, i, X^{*}\right)$.

In other words, assuming $\neg$ bad, in order to detect a difference between $\mathcal{B}$ and $\mathcal{B}^{\prime}, S$ must already have guessed the hidden $X^{*}$ used in the SIM-SO-COM experiment. In particular, since up to that point, oracles $\mathcal{B}$ and $\mathcal{B}^{\prime}$ behave identically, and $S$ can simulate $\mathcal{B}^{\prime}$ internally, $S$ can either extract the hidden $X^{*}$ from the SIM-SO-COM experiment with oracles $\mathcal{R O}$ and $\mathcal{X}$ alone, or not at all. However, since we defined $\mathcal{R O}$ independently and after $\mathcal{X}$, these oracles are independent. Hence, using $\mathcal{R O}$ and $\mathcal{X}$ alone, the view of $S$ is independent of $X^{*}$ unless $S$ explicitly makes a $\mathcal{R O}$-query involving $X^{*}$. Since $X^{*} \in\{0,1\}^{k / 3}$ is uniformly chosen from a suitably large domain, and bad occurs with negligible probability, we get that $S$ 's view is almost statistically independent of $X^{*}$. Consequently, $S$ 's view is almost statistically independent of all $M_{i}^{*}$ with $i \notin I^{*}$. Hence, $S$ can produce out $_{S}=M^{*}$ only with negligible probability.

It remains to prove that bad occurs only negligibly often.

Lemma 3.7. Event bad occurs only with negligible probability.

Proof. We show that any of the events bad $_{\text {coll }}$, badimg $_{\text {img }}$ bad $_{\text {bind }}$ occurs only with negligible probability for any fixed $i, j$. The full claim then can be derived by a union bound over $i, j$, and the individual events. So first fix $i, j$, and note that the functions $\mathcal{R O}\left(\mathrm{Com}^{j}, i, \cdot\right)$ and $\mathcal{R O}\left(\mathrm{Com}, i^{\prime}, \cdot\right)$ are independent as soon as $\operatorname{Com}^{j} \neq \mathrm{Com}$ or $i \neq i^{\prime}$. Hence, for all of the events, we can ignore $\mathcal{R O}$ - and $\mathcal{B}$-queries with a different Com or $i$, and assume that $\mathcal{R O}^{\prime}(\cdot):=\mathcal{R O}\left(\mathrm{Com}^{j}, i, \cdot\right)$ is a fresh random oracle.

bad $_{\text {coll }}$ : Using a birthday bound, we get

$$
\operatorname{Pr}\left[\exists X^{1}, X^{2} \in\{0,1\}^{k / 3}, X^{1} \neq X^{2}: \mathcal{R} \mathcal{O}^{\prime}\left(X^{1}\right)=\mathcal{R} \mathcal{O}^{\prime}\left(X^{2}\right)\right] \leq \frac{\left(2^{k / 3}\right)^{2}}{2^{k}}=2^{-k / 3},
$$

which implies that with large probability, there simply exists no $M_{i}^{j}$ which could raise bad coll.

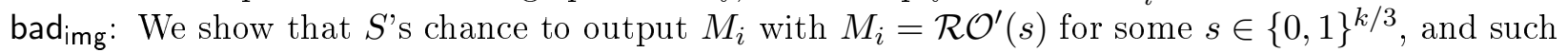
that $X$ has not been queried to $\mathcal{R} \mathcal{O}^{\prime}$-query, is negligible. Now $S$ 's access to the $\mathcal{B}$-oracle can be emulated using an oracle $\mathcal{B}^{\prime}$ that, upon input $Y$, outputs the set of all $X \in\{0,1\}^{k / 3}$ with $\mathcal{R O}^{\prime}(X)=Y$. Without loss of generality, we may further assume that $S$ never queries $\mathcal{B}^{\prime}$ 
with a $Y$ which has been obtained through an explicit $\mathcal{R} \mathcal{O}^{\prime}(X)$-query. (Namely, unless bad coll occurs, which happens only with negligible probability, $\mathcal{B}^{\prime \prime}$ s answer will then be $\{X\}$.)

Hence, whenever $S$ receives an answer $\neq \emptyset$ from $\mathcal{B}^{\prime}$, it has already succeeded in producing an $M_{i}$ with $\mathcal{R \mathcal { O } ^ { \prime }}(X)=M_{i}$ for some $X$, and without querying $\mathcal{R O}^{\prime}(X)$. So without loss of generality, we can assume that $S$ never queries $\mathcal{B}^{\prime}$, and hence only produces such an $M_{i}$ using access to $\mathcal{R O}$ and $\mathcal{X}$ alone. Clearly, $\mathcal{X}$ does not help $S$, since $\mathcal{X}$ and $\mathcal{R O}$ are independent. But since the set of all $Y$ for which $\mathcal{R} \mathcal{O}^{\prime}(X)=Y$ for some $X \in\{0,1\}^{k / 3}$ is sparse in the set of all $Y \in\{0,1\}^{k}$, and $S$ can only make a polynomial number of $\mathcal{R O}$-queries, $S$ 's success in producing such an $M_{i}$ is negligible.

bad $_{\text {bind: }}$ Let $i^{I}$ be the (unique) index for which $\mathcal{B}\left(\left(h_{i}^{j}\right)_{i \in\left[i^{I}\right]}\right)$ outputs $I^{j}$. Without loss of generality, assume that $S$ sets $I^{*}$ after $\mathcal{B}$ first outputs $I^{j}=\mathcal{B}\left(\left(h_{i}^{j}\right)_{i \in\left[i^{I}\right]}\right)$. (Otherwise, $I^{j}=I^{*}$ occurs only with probability $1 /|\mathcal{I}|$, since $I^{j}$ is chosen uniformly and then independent of $I^{*}$.) We can also assume that $\mathrm{Com}^{j}=\mathrm{Com}^{*}$, since otherwise badbind cannot happen by definition. Hence, $S$ first generates a commit transcript $\left(h_{i}^{j}\right)_{i \in\left[i^{I}\right]}$, then receives $I^{j}$ and sends $I^{*}=I^{j}$ to the SIM-SO-COM experiment, and only then receives messages $M_{I^{*}}^{*}$. To achieve bad $_{\text {bind }}$ in this situation, $S$ must find a full transcript $h^{j}$ such that $M_{I^{j}}^{j}=M_{I^{*}}^{*}$. In particular, there is an $i \in I^{j}$ such that $S$ opens the $i$-th commitment in $h^{j}$ to a value $M_{i}^{*}$ which $S$ only sees after the transcript of the commit phase is fixed.

Hence, if $S$ achieves bad $_{\text {bind }}$ with non-negligible probability, we can construct the following PPT machine $A$. A first simulates $S$ to extract $h=h^{j}$, and then rewinds $S$ back to the point before it received $M_{I^{*}}^{*}$. Restarting $S$ with different messages $M_{I^{*}}^{*}$ then yields a transcript $h^{\prime}$ that opens the same commitments as in $h$ to different messages. This contradicts Lemma 3.4.

Taking things together, this shows that $\operatorname{Adv}_{\operatorname{Com}^{*}, \mathcal{M}^{*}, A, S, R}^{\mathrm{sim}-\mathrm{s}}$ is overwhelming for the relation $R(x, y): \Leftrightarrow x=y$, the described $A$, and any PPT $S$. Hence Com* is not SIM-SO-COM secure. It remains to argue that in the described computational world, $\mathcal{X}$ still satisfies property $\mathcal{P}$.

Lemma 3.8. $\mathcal{X}$ satisfies $\mathcal{P}$.

Proof. Assume a PPT adversary $A$ on $\mathcal{X}$ 's property $\mathcal{P}$. Since $\mathcal{X}$ and $\mathcal{P}$ do not query $\mathcal{B}$ or $\mathcal{R O}$, $A$ can do without external oracles $\mathcal{R O}$ and $\mathcal{B}$, and use internal simulations of $\mathcal{R O}$ and $\mathcal{B}$ instead. Using lazy sampling for $\mathcal{R O}$, both simulations can even be made PPT. (This includes $\mathcal{B}$ 's inversion of $\mathcal{R O}$, since we simulate $\mathcal{B}$ and $\mathcal{R O}$ at the same time. We omit the details.)

So without loss of generality, we can assume that $A$ only uses $\mathcal{X}$-queries when interacting with $\mathcal{P}$. Since we assumed that $\mathcal{P}$ holds in the standard model (i.e., without any auxiliary oracles), $A$ 's advantage $\operatorname{Adv}_{\mathcal{P}, \mathcal{X}, A}^{\text {prop }}$ must be negligible.

This concludes the proof of Theorem 3.3 .

The following corollary provides an instantiation of Theorem 3.3 for a number of standard cryptographic primitives.

Corollary 3.9 (Black-box impossibility of non-interactive or perfectly binding SIM-SO-COM). Assume $n$ and $\mathcal{I}$ as in Theorem 3.3. Then no non-interactive or perfectly binding commitment scheme can be proven simulatable under selective openings via a $\forall \exists$ semi-black-box reduction to one or more of the following primitives: one-way functions, one-way permutations, trapdoor one-way permutations, IND-CCA secure public key encryption. 
The corollary is a special case of Theorem 3.3. For instance, to show Corollary 3.9 for oneway permutations, one can use the example $\mathcal{X}$ and $\mathcal{P}$ from above: $\mathcal{X}$ is a random permutation of $\{0,1\}^{k}$, and $\mathcal{P}$ models the one-way experiment with $\mathcal{X}$. Clearly, $\mathcal{X}$ satisfies $\mathcal{P}$, and so we can apply Corollary 3.9. This yields impossibility of relativizing proofs for SIM-SO-COM security from one-way permutations. We get impossibility for $\forall \exists$ semi-black-box reductions since one-way permutations allow embedding, cf. Simon [29], Reingold et al. [27]. The other cases are similar. Note that while it is generally not easy to even give a candidate for a cryptographic primitive in the standard model, it is easy to construct an idealized, say, encryption scheme in oracle form.

Generalizations. First, Corollary 3.9 constitutes merely an example instantiation of the much more general Theorem 3.3. The proof also holds for a relaxation of SIM-SO-COM security considered by Dwork et al. [15, Definition 7.3], where adversary and simulator approximate a function of the message vector.

\subsection{Possibility using non-black-box techniques}

Non-black-box techniques vs. interaction. Theorem 3.3 shows that SIM-SO-COM security cannot be achieved unless one uses non-black-box techniques or interaction. In this section, we will investigate the power of non-black-box techniques to achieve SIM-SO-COM security. As it turns out, for our purposes a concurrently composable zero-knowledge argument system is a suitable non-black-box tool ${ }^{4}$ We stress that the use of this zero-knowledge argument makes our scheme necessarily interactive, and so actually circumvents Theorem 3.3 in two ways: by non-black-box techniques and by interaction. However, from a conceptual point of view, our scheme is "noninteractive up to the zero-knowledge argument." In particular, our proof does not use the fact that the zero-knowledge argument is interactive. (That is, if we used a concurrently composable noninteractive zero-knowledge argument in, say, the common reference string model, our proof would still work.)

The scheme. For our non-black-box scheme, we need an interactive argument system IP with perfect completeness and negligible soundness error, such that IP is zero-knowledge under concurrent composition. We also need a perfectly binding non-interactive commitment scheme Com ${ }^{\mathrm{b}}$. Both these ingredients can be constructed from one-way permutations. To ease presentation, we only describe a bit commitment scheme, which is easily extended (along with the proof) to the multi-bit case.

Scheme 3.10 (Non-black-box commitment scheme ZKCom). Let Com $^{\mathrm{b}}=\left(\mathrm{S}^{\mathrm{b}}, \mathrm{R}^{\mathrm{b}}\right)$ be a perfectly binding non-interactive commitment scheme. Let $I P=(P, V)$ be an interactive argument system for NP which enjoys perfect completeness, has negligible soundness error, and which is zero-knowledge under concurrent composition. Define $\mathrm{ZKCom}=\left(\mathrm{S}^{\mathrm{ZK}}, \mathrm{R}^{\mathrm{ZK}}\right)$ for the following machines $\mathrm{S}^{\mathrm{ZK}}$ and $\mathrm{R}^{\mathrm{ZK}}$ :

- Commitment to bit $b$ :

1. $\mathrm{S}^{\mathrm{ZK}}$ computes $\left(c o m^{0}, d e c^{0}\right) \leftarrow \mathrm{S}^{\mathrm{b}}(b)$ and $\left(\operatorname{com}^{1}, d e c^{1}\right) \leftarrow \mathrm{S}^{\mathrm{b}}(b)$, and sends $\left(c o m^{0}, \operatorname{com}^{1}\right)$ to $\mathrm{R}^{\mathrm{ZK}}$.

2. $\mathrm{S}^{\mathrm{ZK}}$ uses IP to prove to $\mathrm{R}^{\mathrm{ZK}}$ that $\operatorname{com}^{0}$ and $\operatorname{com}^{1}$ commit to the same bit. $5^{5}$

\footnotetext{
${ }^{4}$ We require concurrent composability since the SIM-SO-COM definition considers multiple, concurrent sessions of the commitment scheme.

${ }^{5}$ Formally, the corresponding language $\mathcal{L}$ for IP considers statements $x=\left(\operatorname{com}^{0}, \operatorname{com}^{1}\right)$ and witnesses $w=$ $\left(\operatorname{dec}^{0}, \operatorname{dec}^{1}\right)$ such that $\mathcal{R}(x, w)$ iff $\mathrm{R}^{\mathrm{b}}\left(\operatorname{com}^{0}, \operatorname{dec}^{0}\right)=\mathrm{R}^{\mathrm{b}}\left(\operatorname{com}^{1}, \operatorname{dec}^{1}\right) \in\{0,1\}$.
} 
- Opening:

1. $\mathrm{S}^{\mathrm{ZK}}$ uniformly chooses $j \in\{0,1\}$ and sends $\left(j, d e c^{j}\right)$ to $\mathrm{R}^{\mathrm{ZK}}$.

The security of ZKCom. It is straightforward to prove that ZKCom is a hiding and binding commitment scheme. (We stress, however, that Com ${ }^{\mathrm{b}}$ 's perfect binding property is needed to prove that ZKCom is binding; otherwise, the zero-knowledge argument may become meaningless.) More interestingly, we can also show that ZKCom is SIM-SO-COM secure:

Theorem 3.11 (non-black-box possibility of SIM-SO-COM). Fix any $n$ and $\mathcal{I}$ as in Definition 3.1. Then ZKCom is simulatable under selective openings in the sense of Definition 3.1.

Proof. Assume arbitrary $n, \mathcal{I}, \mathcal{M}, R$, and $A$ as in Definition 3.1. We proceed in games.

Game 0 is the real SIM-SO-COM experiment $\operatorname{ExpZKCom,\mathcal {M},A,R}$ for ZKCom. Define the random variable out $_{0}$ as the output of the experiment, so that

$$
\operatorname{Pr}\left[\operatorname{Exp}_{Z \text { ZKCom, }, \mathcal{M}, A, R}^{\text {sim-so-real }}=1\right]=\operatorname{Pr}\left[\text { out }_{0}=1\right] \text {. }
$$

In Game 1, we interpret the first stage of the experiment as a verifier $V^{*}$ in the sense of Definition 2.5. To this end, we constructively define random variables $x_{i, k}, w_{i, k}, z_{k}^{D}, z_{k}^{V^{*}}$ as follows:

1. sample $M=\left(M_{i}\right)_{i \in[n]} \in\{0,1\}^{n}$ from $\mathcal{M}$,

2. uniformly and independently choose $n$ bits $j_{1}, \ldots, j_{n}$,

3. for all $i \in[n]$ and $j \in\{0,1\}$, compute $\left(\operatorname{com}_{i}^{j}, \operatorname{dec}_{i}^{j}\right) \leftarrow \mathrm{S}^{\mathrm{b}}\left(M_{i}\right)$,

4. define $x_{i, k}=\left(\operatorname{com}_{i}^{0}, \operatorname{com}_{i}^{1}\right), w_{i, k}=\left(\operatorname{dec}_{i}^{0}, \operatorname{dec}_{i}^{1}\right), z_{k}^{V^{*}}=\epsilon$ and $z_{k}^{D}=\left(M,\left(j_{i}, \operatorname{dec}_{i}^{j_{i}}\right)_{i \in[n]}\right)$.

Using this notation, the commitment stage of ExpZKKCom, $\mathcal{M}, A, R$ can be expressed as an interaction of $n$ concurrent instances of prover $\mathrm{P}$ with a suitable verifier $V^{*}$ as in Definition 2.5 ${ }^{6}$ Concretely, we define a verifier $V^{*}$ that, on input $\left(x_{i, k}\right)_{i \in[n]}=\left(\operatorname{com}_{i}^{0}, \operatorname{com}_{i}^{1}\right)_{i \in[n]}$, internally simulates $\operatorname{Exp}_{Z K C \text { Kom }, \mathcal{M}, A, R}^{\text {sim-so-al }}$ up to the point where $A$ outputs $I$. The interactive arguments which show that $\operatorname{com}_{i}^{0}$ and $\operatorname{com}_{i}^{1}$ commit to the same bit are performed concurrently with ( $n$ instances of) a prover $\mathrm{P}$ that gets $x_{i, k}=\left(\operatorname{com}_{i}^{0}, \operatorname{com}_{i}^{1}\right)$ and $w_{i, k}=\left(\operatorname{dec}_{i}^{0}, \operatorname{dec}_{i}^{1}\right)$ as input. Finally, $V^{*}$ outputs out $V^{*}=I$, so that $I$ will be part of the transcript $T_{\mathrm{P}, V^{*}}=\left\langle\mathrm{P}\left(\left(x_{i, k}, w_{i, k}\right)_{i \in[n]}\right), V^{*}\left(\left(x_{i, k}\right)_{i \in[n]}, z_{k}^{V^{*}}\right)\right\rangle$.

We outsource the second stage of the attack into a suitable distinguisher $D$. Concretely, we define a machine $D$ which, on input $z_{k}^{D}=\left(M,\left(j_{i}, d e c_{i}^{j_{i}}\right)_{i \in[n]}\right)$ and a transcript $T_{\mathrm{P}, V^{*}}$ (which contains out $\left._{V^{*}}=I\right)$, simulates out $A \leftarrow A\left(\left(j_{i}, \text { dec }_{i}^{j_{i}}\right)_{i \in I}\right)$ and outputs out $1=R\left(M\right.$, out $\left._{A}\right)$.

This setting is merely a reformulation of ExpZKCom, $\mathcal{M}, A, R$ as a concurrent zero-knowledge argument, so we have that

$$
\operatorname{Pr}\left[\text { out }_{1}=1\right]=\operatorname{Pr}\left[\text { out }_{0}=1\right] .
$$

In Game 2, we use IP's concurrent zero-knowledge property. That is, Game 1 already specifies a PPT verifier $V^{*}$ and a PPT distinguisher $D$, as well as random variables $(x, w), z^{V^{*}}$, and $z^{D}$, as in Definition 2.5. Hence our assumption on IP guarantees that there exists a PPT simulator

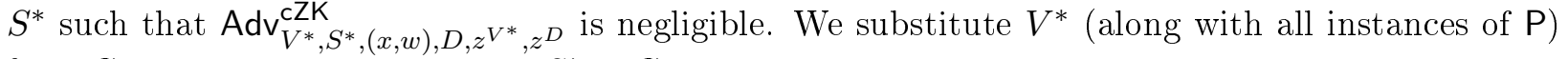
from Game 1 with that simulator $S^{*}$ in Game 2. Note that now, the execution of Game 2 does not require $w_{i, k}=\left(\operatorname{dec}_{i}^{0}, \operatorname{dec}_{i}^{1}\right)$ anymore, but instead only one opening $d e c_{i}^{j_{i}}$ for each argument session. If we let out $t_{2}$ denote $D$ 's output (on input $z_{k}^{D}$ and $o u t_{S^{*}}$ ) in this setting, we get that

$$
\operatorname{Pr}\left[\text { out }_{1}=1\right]-\operatorname{Pr}\left[\text { out }_{2}=1\right]=\operatorname{Adv}_{V^{*}, S^{*},(x, w), D, z^{V^{*}}, z^{D}}^{\text {CZK }}
$$

${ }^{6}$ Note that Definition 2.5 trivially implies security for all distributions on $(x, w), z^{V^{*}}$ and $z^{D}$. Also recall that Definition 2.5 models two different auxiliary inputs $z^{V^{*}}$ (for $V^{*}$ and $S^{*}$ ) and $z^{D}$ (for $D$ ). We emphasize again that this is without loss of generality, cf. the discussion after Definition 2.4 
is negligible.

In Game 3, we use Com ${ }^{\mathrm{b}}$ 's hiding property. Namely, we now change the generation of the $x_{i, k}=\left(\operatorname{com}_{i}^{0}, \operatorname{com}_{i}^{1}\right)$. While we still generate $\operatorname{com}_{i}^{j_{i}}$ as a commitment to $M_{i}$, we now define $\operatorname{com}_{i}^{1-j_{i}}$ as a commitment to $1-M_{i}$, so that $\operatorname{com}_{i}^{0}$ and $\operatorname{com}_{i}^{1}$ are commitments to different bits. Since $\operatorname{dec}_{i}^{1-j_{i}}$ is never used in Game 2, this does not result in a detectable change in D's output. Concretely, we have that

$$
\operatorname{Pr}\left[\text { out }_{3}=1\right]-\operatorname{Pr}\left[\text { out }_{2}=1\right]=\operatorname{Adv}_{\text {Com }^{\mathrm{b}}, A^{\prime}}^{\text {hiding }}
$$

for a suitable adversary $A^{\prime}$ on Com ${ }^{\mathrm{b}}$ 's hiding property, so that $\operatorname{Pr}\left[\right.$ out $\left._{3}=1\right]-\operatorname{Pr}\left[\right.$ out $\left._{2}=1\right]$ is negligible.

To construct Game 4, observe that in Game 3, distinguisher $D$ only needs the openings $\operatorname{dec}_{i}^{j_{i}}$ for $i \in I$ from its input $z_{k}^{D}=\left(M,\left(d e c_{i}^{j_{i}}\right)_{i \in[n]}\right)$. We can exploit this fact as follows. We now generate the commitments $x_{i, k}=\left(\operatorname{com}_{i}^{0}, \operatorname{com}_{i}^{1}\right)$ and openings $\operatorname{dec}_{i}^{j_{i}}$, as well as the $j_{i} \in\{0,1\}$ slightly differently. Concretely, for each message bit $M_{i}$, we first choose a random bit $b_{i}$ and compute $\left(\operatorname{com}_{i}^{0}, \operatorname{dec}_{i}^{0}\right) \leftarrow \mathrm{S}^{\mathrm{b}}\left(b_{i}\right)$ and $\left(\operatorname{com}_{i}^{1}, \operatorname{dec}_{i}^{1}\right) \leftarrow \mathrm{S}^{\mathrm{b}}\left(1-b_{i}\right)$. This modification does not change $S^{*}$ 's view. When $D$ requires an opening $d e c_{i}^{j_{i}}$ (for $i \in I$ ), we define $j_{i}=b_{i} \oplus M_{i}$, so that $d e c_{i}^{j_{i}}$ opens the "right" message $M_{i}$. This does not change the view of $S^{*}$ or $D$, so that we have

$$
\operatorname{Pr}\left[\text { out }_{4}=1\right]=\operatorname{Pr}\left[\text { out }_{3}=1\right] .
$$

The crucial conceptual difference to Game 3 is that now the execution of $D$ requires only knowledge about the message parts $\left(M_{i}\right)_{i \in I}$ selected by $S^{*}$ and not the full message vector $M$.

We can now reformulate Game 4 as an ideal SIM-SO-COM experiment. First, we define a simulator $S$ as follows: first, $S$ prepares bits $b_{i}$ and commitments $\left(\operatorname{com}_{0}^{i}, \operatorname{com}_{1}^{i}\right)$ as in Game 4 and then runs an internal simulation of $S^{*}$ on these commitments. Upon obtaining $I$ from $S^{*}, S$ outputs $I$. Then, upon input $\left(M_{i}\right)_{i \in I}, S$ runs an internal simulation of $A$ on input $\left(j_{i}, \operatorname{dec}_{i}^{j_{i}}\right)_{i \in I}$ for $j_{i}=b_{i} \oplus M_{i}$ as in Game 4. Finally, $S$ outputs out ${ }_{S}=$ out $_{A}$. By construction, the ideal SIM-SO-COM experiment $\operatorname{Exp}_{\mathcal{M}, S, R}^{\text {sim-so-ideal }}$ with this $S$ is only a reformulation of Game 4, so that

$$
\operatorname{Pr}\left[\operatorname{Exp}_{\mathcal{M}, S, R}^{\text {sim-so-ideal }}=1\right]=\operatorname{Pr}\left[\text { out }_{4}=1\right]
$$

Putting things together, we get that

$$
\operatorname{Adv}_{Z K C o m, \mathcal{M}, A, S, R}^{\text {sim-so }}=\operatorname{Pr}\left[\operatorname{Exp}_{Z \text { ZKCom }, \mathcal{M}, A, R}^{\text {sim-so-ral }}=1\right]-\operatorname{Pr}\left[\operatorname{Exp}_{\mathcal{M}, S, R}^{\text {sim-so-ideal }}=1\right]
$$

is negligible, which proves the theorem.

Where is the non-black-box component? Interestingly, the used zero-knowledge argument system IP itself can well be black-box zero-knowledge (where black-box zero-knowledge means that the simulator $S^{*}$ from Definition 2.5 has only black-box access to the next-message function of $V^{*}$ ). The essential fact that allows us to circumvent our negative result Theorem 3.3 is the way we employ IP. Namely, ZKCom uses IP to prove a statement about two given commitments $\left(\mathrm{com}^{0}, \mathrm{com}^{1}\right)$. This proof (or, rather, argument) uses an explicit and non-black-box description of the employed commitment scheme Com ${ }^{\mathrm{b}}$. It is this argument that cannot even be expressed when Com ${ }^{\mathrm{b}}$ makes use of, say, a one-way function given in oracle form.

Generalizations. First, ZKCom can be straightforwardly extended to a multi-bit commitment scheme, e.g., by running several sessions of ZKCom in parallel. Second, ZKCom is SIM-SO-COM secure also against adversaries with auxiliary input $z$ : our proof holds literally, where of course we also require security of $\mathrm{Com}^{\mathrm{b}}$ against non-uniform adversaries. 


\section{An indistinguishability-based definition}

Motivated by the impossibility result from the previous section, we relax Definition 3.1 as follows:

Definition 4.1 (Indistinguishable under selective openings/IND-SO-COM). Let $n=n(k)>0$ be polynomially bounded, and let $\mathcal{I}=\left(\mathcal{I}_{n}\right)_{n}$ be a family of sets such that each $\mathcal{I}_{n}$ is a set of subsets of $[n]$. A commitment scheme $\mathrm{Com}=(\mathrm{S}, \mathrm{R})$ is indistinguishable under selective openings (short IND-SO-COM secure) iff for every PPT n-message distribution $\mathcal{M}$, and every PPT adversary $A$, we have that $\operatorname{Adv}_{\mathrm{Com}, \mathcal{M}, A}^{\text {ind-so }}$ is negligible. Here

$$
\operatorname{Adv}_{\operatorname{Com}, \mathcal{M}, A}^{\text {ind-so }}:=\operatorname{Pr}\left[\operatorname{Exp}_{\mathrm{Com}, \mathcal{M}, A}^{\text {ind-so-real }}=1\right]-\operatorname{Pr}\left[\operatorname{Exp}_{\operatorname{Com}, \mathcal{M}, A}^{\text {ind-so-ideal }}=1\right],
$$

where $\operatorname{Exp}_{\mathrm{Com}, \mathcal{M}, A}^{\text {ind-so-eal }}$ proceeds as follows:

1. sample messages $M=\left(M_{i}\right)_{i \in[n]} \leftarrow \mathcal{M}$,

2. let $A$ (receive) interact concurrently with $n$ instances $\left(\mathrm{S}_{i}\left(\text { commit, } M_{i}\right)\right)_{i \in[n]}$ of $\mathrm{S}$,

3. let $I \in \mathcal{I}$ be $A$ 's output after interacting with the $\mathrm{S}_{i}$,

4. let $A$ (open) interact concurrently with the $|I|$ instances $\left(\mathrm{S}_{i}(\mathrm{open})\right)_{i \in I}$ of $\mathrm{S}$,

5. send the full message vector $M$ to $A$,

6. output A's final output $b$.

On the other hand, Exp $\mathrm{Com}, \mathcal{M}, A$ proceeds as follows:

1. sample messages $M=\left(M_{i}\right)_{i \in[n]} \leftarrow \mathcal{M}$,

2. let $A$ (receive) interact concurrently with $n$ instances $\left(\mathrm{S}_{i}\left(\text { commit, } M_{i}\right)\right)_{i \in[n]}$ of $\mathrm{S}$,

3. let $I \in \mathcal{I}$ be $A$ 's output after interacting with the $\mathrm{S}_{i}$,

4. let $A$ (open) interact concurrently with the $|I|$ instances $\left(\mathrm{S}_{i}(\text { open })\right)_{i \in I}$ of $\mathrm{S}$,

5. sample $M^{\prime} \leftarrow \mathcal{M} \mid M_{I}$, i.e., sample a fresh message vector $M^{\prime}$ from $\mathcal{M}$ with $M_{I}^{\prime}=M_{I}$,

6. send the full vector $M^{\prime}$ to $\mathrm{S}$,

7. output A's final output b.

On the conditioned distribution $\mathcal{M} \mid M_{I}$. We stress that, depending on $\mathcal{M}$, it may be computationally hard to sample $M^{\prime} \leftarrow \mathcal{M} \mid M_{I}$, even if (the unconditioned) $\mathcal{M}$ is PPT. This might seem strange at first and inconvenient when applying the definition in some larger reduction proof. However, there simply seems to be no other way to capture indistinguishability, since the set of opened commitments depends on the commitments themselves. In particular, in general we cannot predict which commitments the adversary wants opened, and then, say, substitute the not-to-be-opened commitments with random commitments. What we chose to do instead is to give the adversary either the full message vector, or an independent message vector which "could be" the full message vector, given the opened commitments. We believe that this is the canonical way to capture secrecy of the unopened commitments under selective openings. We should also stress that it is this definition that turns out to be useful in the context of interactive argument systems, see Section 6

The relation between SIM-SO-COM and IND-SO-COM security. Unfortunately, we (currently) cannot prove that SIM-SO-COM security implies IND-SO-COM security (although this seems plausible, since usually simulation-based definitions imply their indistinguishability-based counterparts). Technically, the reason why we are unable to prove an implication is the conditioned distribution $\mathcal{M} \mid M_{I}$ in the ideal IND-SO-COM experiment, which cannot be sampled from during an (efficient) reduction. 
A relaxation. Alternatively, we could let the adversary predict a predicate $\pi$ of the whole message vector, and consider him successful if $\operatorname{Pr}[b=\pi(M)]$ and $\operatorname{Pr}\left[b=\pi\left(M^{\prime}\right)\right]$ for the alternative message vector $M^{\prime} \leftarrow \mathcal{M} \mid M_{I}$ differ non-negligibly. We stress that our upcoming negative result (as well as the application in Section 6 also applies to this relaxed notion.

\subsection{Impossibility from black-box reductions}

Theorem 4.2 (Black-box impossibility of perfectly binding IND-SO-COM, most general formulation). Let $n=n(k)=2 k$, and let $\mathcal{I}=\left(\mathcal{I}_{n}\right)_{n}$ with $\mathcal{I}_{n}=\{I \subseteq[n]:|I|=n / 2\}$ be the family of all $n / 2$-sized subsets of $[n]$. Let $\mathcal{X}$ be an oracle that satisfies property $\mathcal{P}$ even in presence of an EXPSPACE-oracle. We also demand that $\mathcal{X}$ is computable in EXPSPACE 7 Then, there exists a set of oracles relative to which $\mathcal{X}$ still satisfies $\mathcal{P}$, but no perfectly binding commitment scheme is indistinguishable under selective openings.

Proof. Let $\mathbb{E}=\{0,1\}^{k}$ and $\varepsilon:=.01$. Let $\mathcal{E} \mathcal{X} \mathcal{P} \mathcal{S P} \mathcal{A C E}$ be an EXPSPACE-oracle. We stress that $\mathcal{E X} \mathcal{P} \mathcal{S P} \mathcal{A C E}$ can be used to perform inefficient computations, but $\mathcal{E} \mathcal{X} \mathcal{P} \mathcal{S} \mathcal{A C} \mathcal{E}$ itself never makes oracle queries (e.g., to $\mathcal{X}$ or the oracles $\mathcal{R O}$ and $\mathcal{B}$ presented below). Let $\mathcal{R O}$ be a random function from $\mathbb{E}^{n / 2+1}$ to $\mathbb{E}^{n}$. We write $M \in \mathcal{R O}$ when $M \in \mathbb{E}^{n}$ lies in the range of $\mathcal{R O}$. For $M, M^{\prime} \in \mathbb{E}^{n}$ and $\epsilon>0$, we write $M \equiv_{\varepsilon} M^{\prime}$ iff $M$ and $M^{\prime}$ coincide in at least $\lceil(1-\varepsilon) n\rceil$ components (i.e., iff there exists $R \subseteq[n],|R| \geq\lceil(1-\varepsilon) n\rceil$, with $\left.M_{R}=M_{R}^{\prime}\right)$. To construct our last oracle $\mathcal{B}$, let $B$ be the machine that proceeds as follows.

1. Upon receiving Com as input, check that Com describes a perfectly binding (but not necessarily hiding) commitment scheme (see the discussion after the description of $\mathcal{B}$ ). If not, reject with output $\perp$. If yes, concurrently receive $n$ Com-commitments, indexed by $i \in[n]$.

2. When all commitments are received, output a uniformly chosen $I \in \mathcal{I}$.

3. Engage in $|I|$ concurrent opening phases for the Com-instances with $i \in I$. If all openings are valid (i.e., every Com-receiver instance with $i \in I$ outputs some $M_{i} \neq \perp$ ), then extract the whole message vector $M=\left(M_{i}\right)_{i \in[n]} \in \mathbb{E}^{n}$ from the commitments (this is possible uniquely since Com is perfectly binding). Output the set of all $M^{\prime} \in \mathcal{R O}$ with $M_{I}^{\prime}=M_{I}$ and $M^{\prime} \equiv_{\varepsilon} M$. We should comment on $B$ 's check whether Com is perfectly binding. We want that, for all possible values of $\mathcal{R O}$ and states of $\mathcal{X}$, and for all syntactically allowed commitments, there is at most one message $M_{i}$ to which a commitment can be opened in the sense of Com. Note that by assumption about $\mathcal{X}$, this condition can be checked using $\mathcal{E} \mathcal{X P S P} \mathcal{A C E}$. Concretely, we let $\mathcal{E} \mathcal{X} \mathcal{P} \mathcal{S P} \mathcal{A C E}$ iterate internally over all possible internal states of $\mathcal{X}$ and $\mathcal{B}$, and over all possible random tapes of an honest verifier. $\mathcal{E X P S P} \mathcal{A C E}$ then checks whether a syntactically possible commitment along with two openings to different messages exists. Note that we completely ignore whether or not Com is hiding.

Again, we cannot use $B$ directly, since $B$ is stateful, and black-box separations require stateless oracles. So let $\mathcal{B}$ be the oracle that evaluates $B$ 's next-message function, suitably padded as in the proof of Theorem 3.3. We note that, similarly to Lemma 3.4, we can derive that the perfect binding property of a perfectly binding commitment scheme is preserved by the rewindable formalization in $\mathcal{B}$. In particular, (the transcript of) a commitment phase uniquely determines the only possible opening message.

Lemma 4.3. Let Com* be a perfectly binding commitment scheme (that may use all of the described oracles in its algorithms). Then, Com* is not indistinguishable under selective openings.

\footnotetext{
${ }^{7}$ Examples of such $\mathcal{X}$ are random oracles or ideal ciphers. It will become clearer how we use the EXPSPACE requirement in the proof.
} 
Proof. Consider the $n$-message distribution $\mathcal{M}^{*}$ that samples random elements in the range of $\mathcal{R O}$. (I.e., $\mathcal{M}^{*}$ outputs $\mathcal{R O}(X)$ for a uniformly sampled $X \in \mathbb{E}^{n / 2+1}$.) Consider the following adversary $A$ that relays between the real or ideal IND-SO-COM experiment and oracle $\mathcal{B}$. (Again, we silently assume that $A$ prefixes queries to $\mathcal{B}$ with the respective message history.)

1. Initially, send $\mathrm{Com}^{*}$ to $\mathcal{B}$.

2. Relay the $n$ commitments from the IND-SO-COM experiment to $\mathcal{B}$.

3. Upon receiving $I^{*} \in \mathcal{I}$ from $\mathcal{B}$, send $I^{*}$ to the IND-SO-COM experiment.

4. Upon receiving $\left|I^{*}\right|$ openings from the experiment, relay these openings to $\mathcal{B}$.

5. Upon receiving a challenge message $M$ from the experiment, and a set $S \subseteq \mathbb{E}^{n}$ from $\mathcal{B}$, output out $_{A}=1$ iff $S=\{M\}$.

First, we claim that the probability for $S=\left\{M^{*}\right\}$ is overwhelming, where $M^{*}$ denotes the message vector sampled by the IND-SO-COM experiment. By construction of $\mathcal{B}$, we have $M^{*} \in S$. Furthermore, for any $M^{\prime} \in S$, it must hold that $M^{\prime} \equiv_{\varepsilon} M^{*}$. But for any distinct $X^{1}, X^{2} \in \mathbb{E}^{n / 2+1}$, we have that $\mathcal{R O}\left(X^{1}\right) \equiv_{\varepsilon} \mathcal{R O}\left(X^{2}\right)$ with probability $\left(\begin{array}{c}n \\ \lceil(1-\varepsilon) n\rceil\end{array}\right) /|\mathbb{E}|^{\lceil(1-\varepsilon) n\rceil}$. A union bound over all $M^{\prime} \in \mathcal{R O}$ shows that the probability that there exists an $M^{\prime} \in S, M^{\prime} \neq M^{*}$ is negligible. Hence $S=\left\{M^{*}\right\}$ with overwhelming probability.

Thus, $A$ outputs 1 in the real IND-SO-COM experiment with overwhelming probability, since then $M=M^{*}$. However, in the ideal IND-SO-COM experiment, $M \neq M^{*}$ with overwhelming probability (since for uniformly chosen $M^{*} \in \mathcal{R O}$, the expected number of $M \in \mathcal{R O}$ with $M_{I}=M_{I}^{*}$ is about $|\mathbb{E}|=2^{k}$ ). Consequently, $A$ outputs 1 in the ideal IND-SO-COM experiment only with

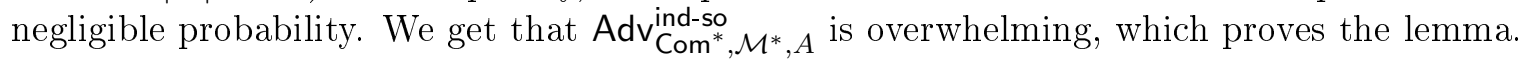

Lemma 4.4. $\mathcal{X}$ satisfies $\mathcal{P}$.

Proof. Consider a PPT adversary $A$ on $\mathcal{X}$ 's property $\mathcal{P}$. Note that $A$ may use $\mathcal{R} \mathcal{O}, \mathcal{B}$, and $\mathcal{E} \mathcal{X} \mathcal{P} \mathcal{P} A \mathcal{E}$ freely. We proceed in games to show that $\operatorname{Adv}_{\mathcal{P}, \mathcal{X}, A}^{\text {prop }}$ is negligible.

Let Game 0 by the original security experiment in which $A$ attacks $\mathcal{X}$ 's property $\mathcal{P}$. We say that a $\mathcal{B}$-query is a commit query (resp. open query) if it finishes the commitment (resp. opening) phase in the corresponding interaction with $B$, such that $\mathcal{B}$ responds with an $I \in \mathcal{I}$ (resp. a set of $M^{\prime} \in \mathcal{R O}$ ). Without loss of generality, we may assume that $A$ never makes commit queries twice, and always makes precisely $p(k)$ open queries for a fixed polynomial $p$. We also assume that for any of $A$ 's open queries, $A$ made a corresponding commit query first 8 Let out $t_{0}$ denote $\mathcal{P}$ 's output in Game 0. By definition, we have

$$
\operatorname{Pr}\left[\text { out }_{0}=1\right]-1 / 2=\operatorname{Adv}_{\mathcal{P}, \mathcal{X}, A}^{\text {prop }} .
$$

In Game $i$ (for $0<i \leq p(k)$ ), we use an oracle $\mathcal{B}_{i}$ instead of oracle $\mathcal{B}$. Here, $\mathcal{B}_{i}$ behaves like $\mathcal{B}$, except that $\mathcal{B}_{i}$ answers each of $A$ 's first $i$ opening queries as follows. Here, $M_{I}=\left(M_{I}\right)_{i \in I}$ denotes the opened messages, as before.

- If all openings are valid, then return the set of all $M^{\prime} \in \mathcal{R O}$ which have been explicitly obtained through $\mathcal{R O}$-queries by $A$ (or $\mathcal{B}_{i}$, in the role of a receiver), and for which $M_{I}^{\prime}=M_{I}$. We stress that oracle $\mathcal{B}_{i}$ does not break a commitment or use internal access to $\mathcal{R O}$ until the $(i+1)$ th open query. Let out $t_{i}$ denote $\mathcal{P}$ 's output in Game $i$. To show that out $t_{i}$ is not significantly affected by our changes, fix an $i$. Let $h$ denote $A$ 's $i$-th open query in Game $i$. Let $S=\mathcal{B}_{i}(h)$ denote the answer $A$ gets in Game $i$, and let $S^{\prime}=\mathcal{B}_{i-1}(h)$ denote the answer that $A$ would have received in

\footnotetext{
${ }^{8}$ In order to violate this assumption, $A$ would have to guess an $I \in \mathcal{I}$ as chosen by $\mathcal{B}$ upon the corresponding commit query. Since $|\mathcal{I}|$ is large, we ignore this possibility.
} 
Game $i-1$. We show in Lemma 4.5 below that $S=S^{\prime}$ except with probability asymptotically smaller than $2^{-3 \varepsilon k}$, so that

$$
\operatorname{Pr}\left[\text { out }_{i}=1\right]-\operatorname{Pr}\left[\text { out }_{i-1}=1\right] \leq 2^{-(\varepsilon / 2) k}
$$

for sufficiently large $k$ and all $i \in[p(k)]$.

Observe that in Game $p(k), \mathcal{B}_{p(k)}$ and $\mathcal{R O}$ can both be simulated efficiently inside $A$. Indeed, $\mathcal{B}_{p(k)}$ only needs knowledge about $A$ 's $\mathcal{R O}$-queries, as well as access to $\mathcal{E} \mathcal{X} \mathcal{P} \mathcal{P} \mathcal{A C E}$ to check whether a given commitment scheme is perfectly binding. Hence,

$$
\operatorname{Adv}_{\mathcal{P}, \mathcal{X}, A^{\prime}}^{\text {prop }}=\operatorname{Pr}\left[\text { out }_{p(k)}=1\right]-1 / 2
$$

for a suitable PPT adversary $A^{\prime}$ that internally simulates $A, \mathcal{R O}$, and $\mathcal{B}_{p(k)}$, and only needs access to $\mathcal{E} \mathcal{X} \mathcal{S} \mathcal{P} \mathcal{A C E}$. By assumption about $\mathcal{X}, \operatorname{Adv}_{\mathcal{P}, \mathcal{X}, A^{\prime}}^{\text {prop }}$ is negligible, and hence so must be $\operatorname{Adv}_{\mathcal{P}, \mathcal{X}, A}^{\text {prop }}$

It remains to prove that, in the situation of Lemma 4.4, $S=S^{\prime}$ with high probability.

Lemma 4.5. In the situation of Lemma 4.4. $\operatorname{Pr}\left[S \neq S^{\prime}\right] \leq 2^{-(\varepsilon / 2) k}$ for sufficiently large $k$.

Combining Lemma 4.6, 4.7, 4.8, and 4.9 below shows Lemma 4.5.

Lemma 4.6. In the situation of Lemma 4.4. $|S| \leq 1$ except with probability at most $q(k) 2^{-k}$ for some polynomial $q$.

Proof. We interpret the whole Game $i$ (including $A, \mathcal{P}, \mathcal{X}, \mathcal{B}_{i}$, and $\mathcal{E} \mathcal{X P S P} \mathcal{A C E}$ ) as a machine $A^{\prime}$ interacting with $\mathcal{R O}$. Note that $A^{\prime}$ may be computationally unbounded, but only makes a polynomial number of $\mathcal{R O}$-queries, at least until $A$ 's $i$-th open query. Let $Q_{\mathcal{R O}}$ denote the set of $\mathcal{R O}$-queries of $A^{\prime}$. Now $|S|>1$ implies that there are $X^{1}, X^{2} \in Q_{\mathcal{R O}}$ with $X^{1} \neq X^{2}$, such that $\mathcal{R O}\left(X^{1}\right), \mathcal{R O}\left(X^{2}\right) \in S$, and so $\mathcal{R O}\left(X^{1}\right)_{I}=\mathcal{R O}\left(X^{2}\right)_{I}$. However, the statistical properties of $\mathcal{R O}$ imply that for any $X^{1}, X^{2} \in Q_{\mathcal{R O}}, \mathcal{R O}\left(X^{1}\right)$ and $\mathcal{R O}\left(X^{2}\right)$ match in at least one component with probability at most $n 2^{-k}$. A union bound over all such pairs shows the claim.

Lemma 4.7. In the situation of Lemma 4.4. $\left|S^{\prime}\right| \leq 1$ except with probability at most $q(k) 2^{-k}$ for some polynomial $q$.

Proof. As in Lemma 4.6, we interpret Game $i$ as a machine $A^{\prime}$ interacting with $\mathcal{R O}$. Again, let $Q_{\mathcal{R O}}$ denote the set of $\mathcal{R O}$-queries of $A^{\prime}$. Now let $\mathbb{X}$ be the set of all $X \in \mathbb{E}^{n / 2+1} \backslash Q_{\mathcal{R O}}$ with $\mathcal{R O}(X)_{I}=M_{I}$. Using, e.g., Chebyshev's inequality, we get $|\mathbb{X}|<2|\mathbb{E}|$, except with probability at most $2^{-k}$. Furthermore, $Q_{\mathcal{R O}}$ contains at most one query $X$ with $\mathcal{R O}(X)_{I}=M_{I}$ except with probability at most $q_{1}(k) 2^{-k}$ for some polynomial $q_{1}$ (with similar reasoning as in Lemma 4.6. Let $\mathbb{X}^{\prime}:=\mathbb{X} \cup\{X\}$ for that $X \in Q_{\mathcal{R O}}$, or $\mathbb{X}^{\prime}:=\mathbb{X}$ if no such $X$ exists. By the preceding discussion, $\left|\mathbb{X}^{\prime}\right| \leq 2 \mathbb{E}$ except with probability $\left(q_{1}(k)+1\right) 2^{-k}$.

Now $\left|S^{\prime}\right|>1$ implies that $X^{1}, X^{2} \in \mathbb{X}^{\prime}$ exist, such that $X^{1} \neq X^{2}$ but $\mathcal{R O}\left(X^{1}\right) \equiv_{\varepsilon} M \equiv_{\varepsilon}$ $\mathcal{R O}\left(X^{2}\right)$, and so $\mathcal{R O}\left(X^{1}\right) \equiv_{2 \varepsilon} \mathcal{R O}\left(X^{2}\right)$. Observe that the values $\mathcal{R O}(X)$ for $X \in \mathbb{X}^{\prime}$ are independent, conditioned only on $\mathcal{R O}(X)_{I}=M_{I}$. For any fixed $X^{1}, X^{2} \in \mathbb{X}^{\prime}$ with $X^{1} \neq X^{2}$, the probability that $\mathcal{R O}\left(X^{1}\right) \equiv_{2 \varepsilon} \mathcal{R O}\left(X^{2}\right)$ is $\left(\begin{array}{c}n / 2 \\ \lceil(1 / 2-2 \varepsilon) n\rceil\end{array}\right) /|\mathbb{E}|^{\lceil(1 / 2-2 \varepsilon) n\rceil}$, which is less than $2^{-3 k-2}$ for sufficiently large $k$. Assuming that $\left|\mathbb{X}^{\prime}\right| \leq 2|\mathbb{E}|=2^{k+1}$, a union bound yields that no such $X^{1}, X^{2}$ exist, and hence $\left|S^{\prime}\right| \leq 1$, except with probability $2^{-k}$. Summing up shows the claim.

Lemma 4.8. In the situation of Lemma 4.4 $S=\emptyset$ but $\left|S^{\prime}\right|=1$ with probability at most $q(k) 2^{-k / 2}$ for some polynomial $q$. 
Proof. Let bad denote the event that $S=\emptyset$ but $S^{\prime}=\left\{M^{\prime}\right\}$ for some $M^{\prime}$, and let $\operatorname{bad}_{j}$ denote the event that bad occurs and $A$ 's $i$-th open query refers to $A$ 's $j$-th commit query. Since $A$ makes only polynomially many $\mathcal{B}_{i}$-queries, there is a polynomial $q_{1}=q_{1}(k)$ and a function $j=j(k)$ such that $\operatorname{Pr}\left[\operatorname{bad}_{j}\right] \geq \operatorname{Pr}[\mathrm{bad}] / q_{1}(k)$.

Consider the machine $A^{\prime}$ that simulates Game $i$ and interacts externally only with oracle $\mathcal{R O}$. Call $I^{1} \in \mathcal{I}$ the answer of $\mathcal{B}_{i}$ to $A^{\prime}$ 's $j$-th commit query. After $A$ submits its $i$-th open query, $A^{\prime}$ rewinds the simulation back to $A$ 's $j$-th commit query, and then restarts with a freshly sampled $I^{2} \in \mathcal{I}$ as $\mathcal{B}_{i}$ 's answer to $A$ 's $j$-th commit query. By bad $_{j, 1}$, resp. $\operatorname{bad}_{j, 2}$, we denote the events that $\operatorname{bad}_{j}$ occurs before, resp. after the rewinding. It is clear that $\operatorname{Pr}\left[\operatorname{bad}_{j, 1}\right]=\operatorname{Pr}\left[\operatorname{bad}_{j, 2}\right]=\operatorname{Pr}\left[\operatorname{bad}_{j}\right]$, but unfortunately, the events $\operatorname{bad}_{j, 1}$ and $\operatorname{bad}_{j, 2}$ may be dependent. We have to work to establish that $\operatorname{bad}_{j, 1}$ and $\operatorname{bad}_{j, 2}$ occur simultaneously with sufficiently large probability. Consider a prefix $E_{j}$ of $A^{\prime}$ 's execution until $A$ 's $j$-th commit query. Given any such $E_{j}$ and a fixed oracle $\mathcal{R O}$, the events $\operatorname{bad}_{j, 1}$ and $\operatorname{bad}_{j, 2}$ are independent and occur with the same probability, so that

$$
\begin{aligned}
\operatorname{Pr}\left[\operatorname{bad}_{j, 1} \wedge \operatorname{bad}_{j, 2}\right]=\sum_{E_{j}, \mathcal{R O}} \operatorname{Pr}\left[\operatorname{bad}_{j, 1} \wedge \operatorname{bad}_{j, 2} \mid\right. & \left.E_{j}, \mathcal{R O}\right] \cdot \operatorname{Pr}\left[E_{j}, \mathcal{R O}\right] \\
=\sum_{E_{j}, \mathcal{R O}} \operatorname{Pr}\left[\operatorname{bad}_{j, 1} \mid E_{j}, \mathcal{R O}\right]^{2} \cdot \operatorname{Pr}\left[E_{j}, \mathcal{R O}\right] \stackrel{(*)}{\geq} & \left(\sum_{E_{j}, \mathcal{R O}} \operatorname{Pr}\left[\operatorname{bad}_{j, 1} \mid E_{j}, \mathcal{R O}\right] \cdot \operatorname{Pr}\left[E_{j}, \mathcal{R O}\right]\right)^{2} \\
& =\operatorname{Pr}\left[\operatorname{bad}_{j, 1}\right]^{2}=\operatorname{Pr}\left[\operatorname{bad}_{j}\right]^{2} \geq \operatorname{Pr}\left[\operatorname{bad}^{2} / q_{1}(k)^{2},\right.
\end{aligned}
$$

where $(*)$ uses that $\sum_{i} c_{i} x_{i}^{2} \geq\left(\sum_{i} c_{i} x_{i}\right)^{2}$ for $c_{i}, x_{i} \geq 0$ with $\sum_{i} c_{i}=1$ by Jensen's inequality.

Let $Q_{\mathcal{R O}, 1}$ denote the set of $A^{\prime}$ 's $\mathcal{R O}$-queries before the rewinding, and let $Q_{\mathcal{R O}, 2}$ denote the set of $A^{\prime}$ 's $\mathcal{R O}$-queries after the rewinding and before $A$ 's $j$-th commit query. The rationale here is that $Q_{\mathcal{R O}, 1}$ are $A$ 's queries in the run related to $I^{1}$, and $Q_{\mathcal{R O}, 2}$ are $A$ 's queries in the run related to $I^{2}$. Note that $Q_{\mathcal{R O}, 1}$ and $Q_{\mathcal{R O}, 2}$ share $A$ 's queries before the $j$-th commitment. We write $\mathcal{R O}\left(Q_{\mathcal{R O}, i}\right)$ for the set of all $\mathcal{R O}(X)$ for $X \in Q_{\mathcal{R O}, i}$.

Now $\operatorname{bad}_{j, 1} \wedge \operatorname{bad}_{j, 2}$ implies that $A$ opens two subsets $M_{I^{1}}$ and $M_{I^{2}}$ message vector $M$ inside the $j$-th commit query, such that there exist $M^{1}, M^{2} \in \mathcal{R O}$ with the following properties:

- $M_{I^{1}}^{1}=M_{I^{1}}$ and $M_{I^{2}}^{2}=M_{I^{2}}$,

- $M^{1} \equiv_{\varepsilon} M \equiv_{\varepsilon} M^{2}$ and hence $M^{1} \equiv_{2 \varepsilon} M^{2}$,

- $M^{1} \notin \mathcal{R O}\left(Q_{\mathcal{R O}, 1}\right)$ and $M^{2} \notin \mathcal{R O}\left(Q_{\mathcal{R O}, 2}\right)$.

We claim that $M^{1}=M^{2}$ with high probability. To see this, let $\mathbb{M}$ be set of all $M^{\prime} \in \mathcal{R O} \backslash \mathcal{R O}\left(Q_{\mathcal{R O}, 1}\right)$ which satisfy $M_{I^{1} \cap I^{2}}^{\prime}=M_{I^{1} \cap I^{2}}$. A simple calculation shows that $m:=\left|I^{1} \cap I^{2}\right| \geq n / 10$ except with probability at most $2^{-k}$ for sufficiently large $k$. Now $|\mathbb{M}|$ 's expected value is, depending on $\left|Q_{\mathcal{R O}, 1}\right|$, at most $|\mathbb{E}|^{n / 2+1-m}$. A Chebyshev bound as in Lemma 4.7 yields that $|\mathbb{M}| \leq|\mathbb{E}|^{n / 2-m+2}$ except with probability at most $q_{2}(k) 2^{-k}$ for some polynomial $q_{2}$. So assume $\left|I^{1} \cap I^{2}\right| \geq n / 10$ and $|\mathbb{M}| \leq|\mathbb{E}|^{n / 2-m+2}$. Then, for any two $M^{1}, M^{2} \in \mathbb{M}$ with $M^{1} \neq M^{2}$, we have $M^{1} \equiv_{2 \varepsilon} M^{2}$ with probability at most $\left(\begin{array}{c}n-m \\ \lfloor 2 \varepsilon n\rfloor\end{array}\right) /|\mathbb{E}|^{n-m-\lfloor 2 \varepsilon n\rfloor}$. A simple calculation and a union bound over all $M^{1}, M^{2} \in \mathbb{M}$ yield that there do not exist $M^{1}, M^{2} \in \mathbb{M}$ with $M^{1} \equiv_{2 \varepsilon} M^{2}$ yet $M^{1} \neq M^{2}$, except with probability at most $q_{3}(k) 2^{-k}$ for some polynomial $q_{3}$. So for the $M^{1}, M^{2}$ guaranteed by $\operatorname{bad}_{j, 1} \wedge \operatorname{bad}_{j, 2}$, either $M^{1}=M^{2}$, or $M^{2} \notin \mathbb{M}$ with high probability.

Now $M^{2} \notin \mathbb{M}$ implies $M^{2}=\mathcal{R O}(X)$ for some $X \in Q_{\mathcal{R O}, 1}$, and $\operatorname{bad}_{j, 2}$ even dictates $X \in Q_{\mathcal{R O}, 1} \backslash$ $Q_{\mathcal{R O}, 2}$. Put differently, $M^{2} \notin \mathbb{M}$ implies that in the execution after the rewinding, $M_{I^{2}}=M_{I^{2}}^{2}$ contains a component of an $\mathcal{R O}$-image $M^{2}$ obtained (independently, since $M^{2} \notin Q_{\mathcal{R O}, 2}$ ) before the rewinding. By symmetry, the probability that this happens equals the probability that $M_{I^{1}}$ contains a component of an $\mathcal{R O}$-image $M^{1}$ queried after the rewinding. However, this essentially 
means that $A^{\prime}$ has guessed a component of the result of an upcoming $\mathcal{R} \mathcal{O}$-query, which can happen with probability at most $q_{4}(k) 2^{-k}$ for some polynomial $q_{4}$ by the statistical properties of $\mathcal{R O}$. We conclude that hence, $M^{2} \in \mathbb{M}$ and so $M^{1}=M^{2}$ except with probability at most $q_{5}(k) 2^{-k}$ for a polynomial $q_{5}$.

Finally, a counting argument shows that $\left|I^{1} \cup I^{2}\right|<n / 2+2$ happens with probability less than $2^{-k}$ for large enough $k$. Summarizing, $\operatorname{bad}_{\text {glue }}:=\operatorname{bad}_{j, 1} \wedge \operatorname{bad}_{j, 2} \wedge\left(M^{1}=M^{2}\right) \wedge\left(\mid I^{1} \cup\right.$ $I^{2} \mid \geq n / 2+2$ ) happens with probability at least $\operatorname{Pr}[\mathrm{bad}]^{2}-q_{6}(k) 2^{-k}$ for some polynomial $q_{6}$. But badglue implies that $A^{\prime}$ has found $J:=I^{1} \cup I^{2}$ with $|J| \geq n / 2+2$, such that there exists an $M^{\prime}:=M^{1}=M^{2} \in \mathcal{R O}$ with $M_{J}^{\prime}=M_{J}$, and $A^{\prime}$ has not obtained $M^{\prime}$ through an explicit $\mathcal{R} \mathcal{O}$-query. Another Chebyshev bound shows that no such $M^{\prime}$ exists, except with probability (over the images $\mathcal{R O} \backslash \mathcal{R O}\left(Q_{\mathcal{R O}, 1} \cup Q_{\mathcal{R O}, 2}\right)$ not queried by $\left.A^{\prime}\right)$ at most $2^{-k}$. Hence, $\operatorname{Pr}\left[\operatorname{bad}_{\text {glue }}\right] \leq 2^{-k}$, so that we finally have $\operatorname{Pr}[\mathrm{bad}] \leq q(k) 2^{k / 2}$ for some polynomial $q$.

Lemma 4.9. In the situation of Lemma 4.4. $|S|=1$ but $S^{\prime}=\emptyset$ with probability at most $2^{-(\varepsilon / 2) k}$ for large enough $k$.

Proof. Again, we interpret the whole Game $i$ (except for $\mathcal{R O}$ ) as a machine $A^{\prime}$ interacting with $\mathcal{R O}$. As in Lemma 4.8, $A^{\prime}$ waits for $A^{\prime}$ 's $i$-th open query $M_{I}$, and then rewinds the whole game back to $A^{\prime}$ 's $j$-th commit query. Again, $A^{\prime}$ re-samples an $I \leftarrow \mathcal{I}$ as a fresh answer to $A$ 's $j$-th commit query, in the hope that $A$ opens $M_{I}$ in the $i$-th open query. However, this time $A^{\prime}$ repeats this process $p(k)$ times for a suitable number $p(k)$ to be determined later. Let $S^{\ell}$ and $I^{\ell}$ denote the values of $I$ and $S$ from the $\ell$-th rewinding.

Now fix random tapes for all machines simulated inside $A^{\prime}$, and fix an $\mathcal{R O}$. This means that the only randomness during the execution of $A^{\prime}$ comes from the choice of the $I^{\ell}$. Let bad denote the event that $|S|=1$ but $S^{\prime}=\emptyset$, and let $\operatorname{bad}_{j}$ denote the event that bad occurs and $A$ 's $i$-th open query refers to $A$ 's $j$-th commit query. Since $A$ makes only polynomially many $\mathcal{B}_{i}$-queries, there is a polynomial $q=q(k)$ and a function $j=j(k)$ such that $\operatorname{Pr}\left[\operatorname{bad}_{j}\right] \geq \operatorname{Pr}[\mathrm{bad}] / q(k)$, where the probability is only over $I \in \mathcal{I}$.

Suppose that $\operatorname{Pr}[\mathrm{bad}]>2^{-(\varepsilon / 2) k}$ for contradiction, so that $\operatorname{Pr}\left[\operatorname{bad}_{j}\right]>2^{-\varepsilon k}$ for large enough $k$.

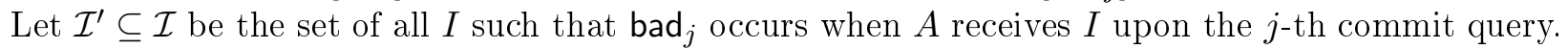
Note that $\mathcal{I}^{\prime}$ is well-defined, since we fixed all randomness except for $I$. Assume first that there exists a subset $B \subseteq[n]$ of size $|B|>\lfloor\varepsilon n\rfloor$ with $\operatorname{Pr}\left[I \in \mathcal{I}^{\prime} \wedge i \in I\right]<2^{-2 \varepsilon k}$ for all $i \in B$, where the probability is over $I \in \mathcal{I}$. We have $\operatorname{Pr}[I \cap B=\emptyset]=\left(\begin{array}{c}\lceil(1-\varepsilon) n\rceil \\ n / 2\end{array}\right) /\left(\begin{array}{c}n \\ n / 2\end{array}\right) \leq 2^{-\varepsilon n}=2^{-2 \varepsilon k}$, so

$$
\begin{aligned}
2^{-\varepsilon k}-2^{-2 \varepsilon k} \leq \operatorname{Pr}\left[I \in \mathcal{I}^{\prime}\right]-\operatorname{Pr}[I \cap B=\emptyset] \leq \operatorname{Pr}\left[I \in \mathcal{I}^{\prime} \wedge I \cap B \neq \emptyset\right] & \\
& \leq \sum_{i \in B} \operatorname{Pr}\left[I \in \mathcal{I}^{\prime} \wedge i \in I\right]<n \cdot 2^{-2 \varepsilon k}
\end{aligned}
$$

creates a contradiction for sufficiently large $k$. Hence, no such $B$ exists, and so there must be a subset $R \subseteq[n]$ of size $|R| \geq\lceil(1-\varepsilon) n\rceil$ such that $\operatorname{Pr}\left[I \in \mathcal{I}^{\prime} \wedge i \in I\right] \geq 2^{-2 \varepsilon k}$ for all $i \in R$.

Our goal is now to use $A^{\prime}$ to extract $M_{R}$ with high probability. To this end, we first finish our description of $A^{\prime}$. Let $L$ denote the set of all $\ell \in[p(k)]$ for which $\operatorname{bad}_{j}$ occurs in the $\ell$-th rewinding. After $p(k):=2^{8 \varepsilon k}$ rewindings, $A^{\prime}$ outputs $M_{J}$, where $J=\bigcup_{\ell \in L} I^{\ell}$ is the union of all successfully extracted partial message subsets. For $\ell \in L$, we have $\left|S^{\ell}\right|=1$ by definition of $\operatorname{bad}_{j}$, so say $S^{\ell}=\left\{M^{\ell}\right\}$. By definition, $M^{\ell}$ has been obtained by $A^{\prime}$ through an explicit $\mathcal{R} \mathcal{O}$-query, and we have $M_{I^{\ell}}^{\ell}=M_{I^{\ell}}$ for the message vector $M$ inside $A^{\prime}$ 's $j$-th commit query. Similar to Lemma 4.6, all components of all $\mathcal{R O}$-images obtained by $A^{\prime}$ are pairwise distinct, except with probability at most $2^{-k / 2}$ for large enough $k$. As in Lemma 4.8, we can show that all the $\mathcal{R} \mathcal{O}$-images $M^{\ell}$ are identical, 
except with probability $2^{-k / 2}$ for sufficiently large $k$. Thus, there exists one single $M^{\prime} \in \mathcal{R O}$ with $M_{J}^{\prime}=M_{J}$. Now note that the $I^{\ell}$ are independent. Hence, a Chebyshev bound shows that for each fixed $i \in R$, there is an $I^{\ell} \in L \subseteq \mathcal{I}^{\prime}$ with $i \in I^{\ell}$, except with probability at most $2^{-6 \varepsilon k}$. A union bound over all $i \in R$ yields $R \subseteq J$ except with probability at most $2^{-5 \varepsilon k}$ for large enough $k$. So, except with probability $2^{-6 \varepsilon k}+2^{k / 2}<\operatorname{Pr}$ [bad], $A^{\prime}$ shows the existence of an $M^{\prime} \in \mathcal{R O}$ with $M_{J}^{\prime}=M_{J}$ for $|J| \geq\lceil(1-\varepsilon) n\rceil$, such that $M^{\prime} \equiv_{\varepsilon} M$. Since $M_{I^{\ell}}^{\prime}=M_{I^{\ell}}$ for any $I^{\ell} \in L$, this contradicts bad $_{j}$ and thus bad. Hence, our assumption on $\operatorname{Pr}$ [bad] must have been incorrect, and we have proved the lemma.

Combining Lemma 4.3 and Lemma 4.4 shows Theorem 4.2.

We stress that the requirement in Theorem 4.2 on $\mathcal{X}$ is a rather mild one. For instance, random oracles are one-way even against computationally unbounded adversaries, as long as the adversary makes only a polynomial number of oracle queries. Hence, an EXPSPACE-oracle (which itself does not perform oracle queries) is not helpful in breaking a random oracle. So similarly to Corollary 3.9. we get for concrete choices of $\mathcal{X}$ and $\mathcal{P}$ :

Corollary 4.10 (Black-box impossibility of perfectly binding IND-SO-COM). Let $n$ and $\mathcal{I}$ as in Theorem 4.2. Then no perfectly binding commitment scheme can be proved indistinguishable under selective openings via a $\forall \exists$ semi-black-box reduction to one or more of the following primitives: oneway functions, one-way permutations, trapdoor one-way permutations, IND-CCA secure public key encryption.

Generalizations. Again, Corollary 4.10 constitutes merely an example instantiation of the much more general Theorem 4.2. We stress, however, that the proof for Theorem 4.2 does not apply to "almost-perfectly binding" commitment schemes such as the one from Naor [24]. (For instance, for such schemes, $\mathcal{B}$ 's check that the supplied commitment scheme is binding might tell something about $\mathcal{X}$.)

\subsection{Statistically hiding schemes are secure}

Fortunately, things look different for statistically hiding commitment schemes:

Theorem 4.11 (Statistically hiding schemes are IND-SO-COM secure). Fix arbitrary $n$ and $\mathcal{I}$ as in Definition 4.1, and let $\mathrm{Com}=(\mathrm{S}, \mathrm{R})$ be a statistically hiding commitment scheme. Then Com is indistinguishable under selective openings in the sense of Definition 4.1.

Proof. Fix an $n$-message distribution $\mathcal{M}$ and a PPT adversary $A$ on the SIM-SO-COM security of Com. We proceed in games.

Game -1 is the real IND-SO-COM experiment $\operatorname{Exp}_{\mathrm{Com}, \mathcal{M}, A}^{\text {ind }, \text {. Let } \text { out }}-1$ denote the output of the experiment, so that we have

$$
\operatorname{Pr}\left[\operatorname{Exp}_{\text {Com }, \mathcal{M}, A}^{\text {ind-so-real }}=1\right]=\operatorname{Pr}\left[\text { out }_{-1}=1\right] .
$$

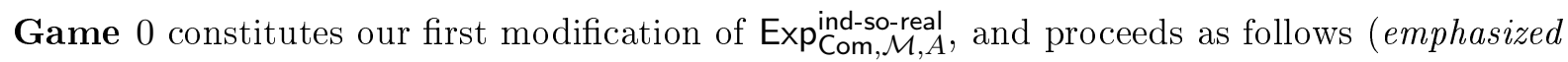
steps are different from $\left.\operatorname{Exp}_{\mathrm{Com}, \mathcal{M}, A}^{\text {ind-so-real }}\right)$ :

1. sample messages $M=\left(M_{i}\right)_{i \in[n]} \leftarrow \mathcal{M}$,

2. let $A$ (receive) interact concurrently with $n$ instances $\left(\mathrm{S}_{i}\left(\text { commit, } M_{i}\right)\right)_{i \in[n]}$ of $\mathrm{S}$,

3. let $I \in \mathcal{I}$ be $A$ 's output after interacting with the $\mathrm{S}_{i}$, 
4. for every $i \in I$, set the $i$-th sender's state to the output of procedure $\operatorname{AltDec}\left(H_{i}, M_{i}\right)$ (described below), where $H_{i}$ denotes the exchanged messages during the commit phase of the $i$-th Com instance,

5. let $A$ (open) interact concurrently with the $|I|$ instances $\left(\mathrm{S}_{i} \text { (open) }\right)_{i \in I}$ of $\mathrm{S}$,

6. send the full message vector $M$ to $A$,

7. output $A$ 's final output $b$.

The (in general inefficient) procedure AltDec takes as input a history $H_{i}$ of exchanged messages in the commit phase and a message $M_{i}$. We call a random tape $t$ for $\mathrm{S}$ consistent with $H_{i}$ and $M_{i}$ iff $\mathrm{S}$ (commit, $M_{i}$ ) (with random tape $t$ ) produces the sender's messages in $H_{i}$ when receiving the respective receiver's replies in $H_{i}$. Let $T_{H_{i}, M_{i}}$ denote the set of all random tapes $t$ for $\mathrm{S}$ which are consistent with $H_{i}$ and $M_{i}$. Now $\operatorname{AltDec}\left(H_{i}, M_{i}\right)$ samples uniformly a random tape $t$ from $T_{H_{i}, M_{i}}$ and returns the state of $\mathbf{S}$ with random tape $t$ and after an interaction according to $H_{i}$. If $T_{H_{i}, M_{i}}=\emptyset$, then AltDec returns $\perp$ (and Game 0 aborts with output 0 ). In other words, AltDec returns the state of a sender $\mathrm{S}$ with initial input $M_{i}$, conditioned on the transcript $T_{i}$ of the commit phase.

In Game 0, AltDec will never return $\perp$ (since AltDec is invoked with a transcript $H_{i}$ that has actually been produced as a commit phase to $M_{i}$ ). Moreover, the view of the adversary is not altered by re-sampling the internal state of the sender, conditioned on all previous actions, as AltDec does. Hence, we have

$$
\operatorname{Pr}\left[\text { out }_{0}=1\right]=\operatorname{Pr}\left[\text { out }_{-1}=1\right]
$$

for the output out $t_{0}$ of the experiment in Game 0.

We describe Game $j$ (for $j \in[n]$ ). Game $j$ is identical to Game 0, except for step 2:

$2^{*}$. let $A$ (receive) interact concurrently with $n$ instances $\left(\mathrm{S}_{i}\left(\text { commit, } M_{i}^{*}\right)\right)_{i \in[n]}$ of $\mathrm{S}$, where we set $M_{i}^{*}=0^{k}$ for $i \leq j$ and $M_{i}^{*}=M_{i}$ for $j>i$,

Obviously, for $j=0$ we would get Game 0 . Note that only difference between Game $j-1$ and Game $j$ is the commitment to $M_{j}$. In fact, we can now construct an adversary $A^{\prime}$ on Com's statistical hiding property. $A^{\prime}$ first uniformly chooses $j \in[n]$, then simulates Game $j-1$, but picks $M_{j}$ and $0^{k}$ as challenge messages for its own experiment $\operatorname{Exp}_{\mathrm{Com}, A^{\prime}}^{\text {hiding } b}$ The $j$-th commitment (to either $M_{j}$ or $\left.0^{k}\right)$ is performed through the experiment. $\operatorname{Exp}_{\mathrm{Com}, A^{\prime}}^{\text {hiding } 0}$ is then a perfect simulation of Game $j-1$, and Exp $\mathrm{Com}_{\mathrm{Com}, A^{\prime}}^{\text {hing } 1}$ perfectly simulates Game $j$. (However, we stress that $A^{\prime}$ is inherently unbounded: $A^{\prime}$ must run procedure AltDec.) We get that

$$
\operatorname{Pr}\left[\text { out }_{n}=1\right]-\operatorname{Pr}\left[\text { out }_{0}=1\right]=n \cdot \operatorname{Adv}_{\text {Com, } A^{\prime}}^{\text {hiding }}
$$

must be negligible, which proves that

$$
\operatorname{Pr}\left[\operatorname{Exp}_{\operatorname{Com}, \mathcal{M}, A}^{\text {ind-so-real }}=1\right]-\operatorname{Pr}\left[\text { out }_{n}=1\right]
$$

is negligible.

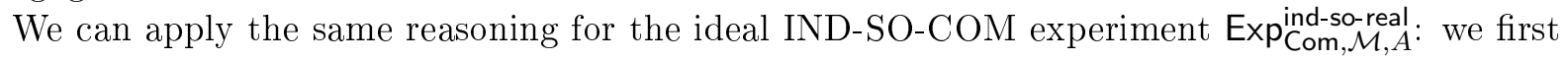
construct the openings using the commit transcripts $H_{i}$ and the target messages $M_{i}$ alone as in Game 0 above. Then we change the actual commitments to commitments to $0^{k}$, as in Game 1 up to Game $n$ above. At this point, the modified ideal experiment first samples $M \leftarrow \mathcal{M}$ and then $M^{\prime} \leftarrow \mathcal{M} \mid M_{I}$, but never uses $M$. Hence we can sample $M^{\prime} \leftarrow \mathcal{M}$ in the first place without changing $A$ 's view. But this is then exactly Game $n$ from above, so that we get that

$$
\operatorname{Pr}\left[\operatorname{Exp}_{\text {Com }, \mathcal{M}, A}^{\text {ind-so-ideal }}=1\right]-\operatorname{Pr}\left[\text { out }_{n}=1\right]
$$

is negligible. Hence $\operatorname{Adv}_{\mathrm{Com}, \mathcal{M}, A}^{\text {ind-so }}$ is negligible as well, which shows the theorem. 
We stress that the proof of Theorem 4.11 also holds (literally) in case $A$ and/or $\mathcal{M}$ gets an additional auxiliary input $z$.

\section{Application to adaptively secure encryption}

Motivation and setting. Taking up the motivation of Damgård [11, we consider the setting of an adversary $A$ that may corrupt, in an adaptive manner, a subset of a set of parties $P_{1}, \ldots, P_{n}$. Assume that for all $i$, the public encryption key $p k_{i}$ with which party $P_{i}$ encrypts outgoing messages, is publicly known. Suppose further that $A$ may corrupt parties based on all public keys and all so far received ciphertexts. When $A$ corrupts $P_{i}, A$ learns $P_{i}$ 's internal state and history, in particular $A$ learns the randomness used for all of that party's encryptions, and its secret key $s k_{i}$. We assume the following:

1. The number of parties is $n=2 k$ for the security parameter $k$,

2. It is allowed for $A$ to choose at some point a subset $I \subseteq[n]$ of size $n / 2$ and to corrupt all these $P_{i}(i \in I)$.

3. We can interpret the used encryption scheme as a (non-interactive, hiding and binding) commitment scheme Com $=(\mathrm{S}, \mathrm{R})$ in the following sense: $\mathrm{S}(M)$ generates a fresh public key $p k$ and outputs a commitment $c o m=(p k$, Enc $(p k, M ; r))$ and an opening $d e c=(M, r)$. Here Enc denotes the encryption algorithm of the encryption scheme, and $r$ denotes the randomness used while encrypting $M$. Verification of $(c o m, d e c)=(p k, C, M, r) \operatorname{checks~that~} \operatorname{Enc}(p k, M ; r)=C$. Note that the third assumption does not follow from the scheme's correctness. Indeed, correctness implies only that honestly generated $(p k, M)$ are committing. However, there are schemes for which it is easy to come up with fake public keys and ciphertexts (i.e., fake commitments) which are computationally indistinguishable from honestly generated commitments, but can be opened in arbitrary ways. Prominent examples of such schemes are non-committing encryption schemes [7, [4, 8, 12, 10], which however generally contain interaction from time to time and are comparatively inefficient.

Application of our impossibility results. Attacks in this setting cannot be easily simulated in the sense of, e.g., Canetti et al. [7]: such a simulator would in particular be able to simulate openings (in the sense of Com, i.e., openings of ciphertexts). Hence, this would imply a simulator for Com in the sense of SIM-SO-COM security (Definition 3.1). Now from Corollary 3.9 we know that the construction and security analysis of such a simulator requires either a very strong computational assumption, or fundamentally non-black-box techniques. Even worse: if Com is perfectly binding 9 then Corollary 4.10 shows that not even secrecy in the sense of Definition $4.1^{10}$ can be proved in a black-box way. On top of that, we cannot hope to use our (non-black-box) SIM-SO-COM secure scheme ZKCom to construct an encryption scheme in a non-black-box way, since ZKCom's commitment phase is inherently interactive.

We stress that these negative results only apply if encryption really constitutes a (binding) commitment scheme in the above sense. In fact, e.g., [7] construct a sophisticated non-committing (i.e., non-binding) encryption scheme and prove simulatability for their scheme. Our results show that such a non-committing property is to a certain extent necessary.

\footnotetext{
${ }^{9}$ in the presence of non-uniform adversaries, this is already implied by the fact that the scheme is non-interactive and computationally binding

${ }^{10}$ in the context of encryption, Definition 4.1 would translate to a variant of indistinguishability of ciphertexts
} 


\section{Application to zero-knowledge proof systems}

\subsection{Graph 3-coloring is composable in parallel}

Outline. Dwork et al. [15] have considered the applications of SIM-SO-COM secure commitment schemes to zero-knowledge protocols, in particular to the graph 3-coloring interactive proof system G3C of Goldreich et al. [20]. Concretely, [15, Theorem 7.6] states that G3C, when instantiated with a SIM-SO-COM secure commitment scheme, retains a relaxed zero-knowledge property called " $S(V, T, D)$ zero-knowledge" under parallel composition. $S(V, T, D)$ zero-knowledge is a variant of zero-knowledge in which the simulator $S$ may depend on the verifier $V$, on the distinguisher $T$ between real and simulated transcript, and on the considered message distribution $D$. Unfortunately, [15] could not give a SIM-SO-COM secure commitment scheme to implement their theorem.

Using our scheme ZKCom, we can instantiate and in fact generalize [15, Theorem 7.6]. Concretely, using a refined analysis and the specific structure of ZKCom, we show that G3C, when implemented with ZKCom, is zero-knowledge under parallel composition. This is surprising in light of the negative composability results Goldreich and Krawczyk [19], Canetti et al. [9]. Similar to Barak [1], we use non-black-box techniques to circumvent known impossibilities.

Commit-choose-open protocols. We can actually prove parallel composability of a larger class of "commit-choose-open" style interactive argument systems:

Definition 6.1 (Commit-choose-open (CCO) protocol). Let $\mathrm{IP}=(\mathrm{P}, \mathrm{V})$ be an interactive argument system for an NP-language $\mathcal{L}$ with witness relation $\mathcal{R}$. Let $n=n(k)>0$ be polynomially bounded, and let $\mathcal{I}=\left(\mathcal{I}_{n}\right)_{n}$ be a family of sets such that each $\mathcal{I}_{n}$ is a set of subsets of $[n]$. We say that IP is a commit-choose-open (CCO) protocol (that uses commitment scheme Com) if the following holds. First, we require that IP is of the following form:

1. $\mathrm{P}$, upon input $(x, w)$ with $x \in \mathcal{L}$ and $\mathcal{R}(x, w)$, selects $n$ messages $\left(M_{i}\right)_{i \in[n]}$,

2. $\mathrm{P}$ engages in $n$ instances of Com to commit to the $M_{i}$ at $\mathrm{R}$,

3. $\mathrm{V}$, upon input $x$, chooses a subset $I \in \mathcal{I}_{n}$ and sends $I$ to $\mathrm{P}$,

4. $\mathrm{P}$ opens all Com-commitments to $M_{i}$ with $i \in I$,

5. $\mathrm{V}$ accepts if the openings are valid and if the opened values satisfy some fixed relation specified by the protocol.

Second, we require that the messages $\left(M_{i}\right)_{i \in I}$ opened by $\mathrm{P}$ in the third step are uniform and independent values over their respective domain. (In particular, $\left(M_{i}\right)_{i \in I}$ can be efficiently sampled without knowing a witness $w$.)

It is easy to verify that the mentioned graph 3-coloring protocol G3C [20] is a CCO protocol. Also, trivially, the parallel composition of many instances of a CCO protocol is again a CCO protocol. In particular, in the following, we will for simplicity only talk about a single CCO protocol, while one should actually have the parallel composition of, e.g., G3C in mind.

Auxiliary-input SIM-SO-COM security. We will prove that any CCO protocol, when using a commitment scheme which is simulatable under selective openings, is black-box zero-knowledge. To this end, we need a refinement of SIM-SO-COM security, which captures auxiliary input and an order of quantifiers as in the zero-knowledge definition.

Definition 6.2 (AI-SIM-SO-COM). In the situation of Definition 3.1, we say that Com is AI-SIM$S O-C O M$ secure, iff for every PPT adversary $A$, there exists a PPT simulator $S$, such that for every PPT relation $R$, every PPT n-message distribution $\mathcal{M}$, and all auxiliary inputs $z^{\mathcal{M}}=\left(z_{k}^{\mathcal{M}}\right)_{k \in \mathbb{N}} \in$ 
$\left(\{0,1\}^{*}\right)^{\mathbb{N}}, z^{A}=\left(z_{k}^{A}\right)_{k \in \mathbb{N}} \in\left(\{0,1\}^{*}\right)^{\mathbb{N}}$, and $z^{R}=\left(z_{k}^{R}\right)_{k \in \mathbb{N}} \in\left(\{0,1\}^{*}\right)^{\mathbb{N}}$, we have that the advantage $\operatorname{Adv}_{\mathrm{Com}, \mathcal{M}, A, S, R, z^{\mathcal{M}}, z^{A}, z^{R}}^{\text {sim-so }}$ is negligible. Here, $\operatorname{Adv}_{\mathrm{Com}, \mathcal{M}, A, S, R, z^{\mathcal{M}}, z^{A}, z^{R}}^{\text {sim-so }}$ is defined as $\operatorname{Adv}_{\text {Com }, \mathcal{M}, A, S, R}^{\text {sim-so }}$, with the following differences:

- $\mathcal{M}$ gets additional input $z^{\mathcal{M}}$,

- $A$ and $S$ get additional input $z^{A}$, and

- $R$ gets additional input $z^{R}$.

We claim that our scheme ZKCom from Section 3.2 satisfies Definition 6.2, To see this, recall that the simulator $S$ constructed in the proof of Theorem 3.11 works also in the presence of auxiliary input. Furthermore, $S$ does not depend on $\mathcal{M}$ and $R$. However, since $\mathcal{M}, S, A$, and $R$ all receive an auxiliary input in the AI-SIM-SO-COM experiment, we must demand that the commitment schemes $\mathrm{Com}^{\mathrm{b}}$ and $\mathrm{Com}^{\mathrm{h}}$ against non-uniform adversaries. We get:

Theorem 6.3 (ZKCom is AI-SIM-SO-COM). Suppose that there exist one-way permutations secure against non-uniform adversaries. Then our commitment scheme ZKCom from Section 3.2 can be instantiated such that ZKCom achieves AI-SIM-SO-COM security for arbitrary $n, \mathcal{I}$.

The following theorem is a generalization of Dwork et al. [15, Theorem 7.6]:

Theorem 6.4 (AI-SIM-SO-COM implies zero-knowledge). Let IP $=(\mathrm{P}, \mathrm{V})$ be a CCO protocol that uses a commitment scheme Com. If Com is AI-SIM-SO-COM secure (for $n$ and $\mathcal{I}$ as used in IP), then IP is zero-knowledge in the sense of Definition 2.4.

Proof. Assume $V^{*},(x, w), D, z^{V^{*}}$, and $z^{D}$ as in Definition 2.4. We will construct a suitable PPT simulator $S^{*}$. Since IP is a CCO protocol, we can immediately use the AI-SIM-SO-COM security of Com. To this end, we define an adversary $A$, a message distribution $\mathcal{M}$, a relation $R$, and auxiliary inputs $z^{A}$ and $z^{R}$ as in Definition 6.2

Concretely, define $z^{\mathcal{M}}=(x, w)$ and let $\mathcal{M}$ be the PPT $n$-message distribution that is induced by $\mathrm{P}$ on input $(x, w)$. Furthermore, let $z^{A}=\left(x_{k}, z^{V^{*}}\right)$ and let $A=V^{*}$, except that $A$ finally outputs a transcript of its conversation. We hence have out $A=\left\langle\mathrm{P}\left(x_{k}, w_{k}\right), V^{*}\left(x_{k}, z_{k}^{V^{*}}\right)\right\rangle$. Finally, set $z^{R}=z^{D}$ and $R\left(M\right.$, out,$\left.z^{R}\right)=D\left(\right.$ out,$\left.z^{R}\right)$, such that $R$ outputs exactly what $D$ outputs on real transcripts as in Definition 2.4. Now Definition 6.2 guarantees that there exists a PPT machine $S$ such that

$$
\begin{aligned}
\operatorname{Pr}\left[R\left(M, \text { out }_{A}, z^{R}\right)=1\right]- & \operatorname{Pr}\left[R\left(M, \text { out }_{S}, z^{R}\right)=1\right] \\
= & \operatorname{Pr}\left[D\left(\left\langle\mathrm{P}\left(x_{k}, w_{k}\right), V^{*}\left(x_{k}, z_{k}^{V^{*}}\right)\right\rangle, z^{D}\right)=1\right]-\operatorname{Pr}\left[D\left(\text { out }_{S}, z^{D}\right)=1\right]
\end{aligned}
$$

is negligible, where out $t_{S}$ denotes the final output of $S$ in the ideal AI-SIM-SO-COM experiment. Note that out $_{S}$ is still obtained through an interactive experiment that in particular requires knowledge about $M$ and hence the witness $w$. However, the only information $S$ actually receives about the message vector $M$ is the subset $M_{I}=\left(M_{i}\right)_{i \in I}$. Since IP is a CCO protocol in the sense of Definition 6.1, $M_{I}$ is statistically independent of $(x, w)$. Hence we can construct the following machine $S^{*}$ which has oracle access to $A=V^{*}$. Namely, $S^{*}$ internally simulates $S$ (and relays to $S^{*}$ its own oracle access to $A$ ). As soon as $S$ outputs a set $I, S^{*}$ answers with a uniformly and independently sampled set $\left(M_{i}\right)_{i \in I}$. Note that $S^{*}$ no longer takes part in a AI-SIM-SO-COM experiment, but instead works with input $z^{A}=\left(x_{k}, z^{V^{*}}\right)$ and oracle access to $V^{*}$ alone. By the CCO property of $\mathrm{IP}$, we obtain

$$
\operatorname{Pr}\left[D\left(\text { out }_{S}, z^{D}\right)=1\right]=\operatorname{Pr}\left[D\left(S^{*}\left(x_{k}, z^{V^{*}}, z^{D}\right)=1\right],\right.
$$

and hence, putting things together shows that $\operatorname{Adv}_{V^{*}, S^{*},(x, w), D, z^{V^{*}}, z^{D}}^{\mathrm{ZK}}$ is indeed negligible. 
Observing that the mentioned graph 3-coloring protocol G3C from Goldreich et al. [20] is a CCO protocol, and that the set of CCO protocols are closed under parallel composition we get:

Corollary 6.5 (G3C is composable in parallel). The graph 3-coloring protocol G3C, when implemented with our commitment scheme ZKCom, is zero-knowledge, even under parallel composition.

What our positive results do not imply (and what our negative results do imply). We emphasize as well that our results do not imply that there are no, in the terminology of [15], "magic functions." In order to prove non-existence of magic functions with [15, Theorem 5.1], one would have to find a non-interactive SIM-SO-COM secure commitment scheme. Our negative result Theorem 3.3 states that this will not be possible with black-box reductions to standard assumptions.

\subsection{IND-SO-COM security and witness indistinguishability}

Outline. A natural question is whether IND-SO-COM security, our relaxation of SIM-SO-COM security, provides a reasonable fallback for SIM-SO-COM security. Now first, our results show that even when using IND-SO-COM secure schemes, we cannot rely on perfectly binding commitment schemes because of Theorem 4.2. For many interesting interactive proofs (and in particular the mentioned graph 3-coloring protocol G3C), this unfortunately means that the proof system degrades to an argument system. But, assuming we are willing to pay this price, what do we get from INDSO-COM security?

The answer is "essentially witness indistinguishability," as we will argue in a minute. Essentially, any commitment scheme which satisfies (a slight variation of) IND-SO-COM security can be used to implement commit-choose-open style interactive argument systems. The resulting argument system will be witness-indistinguishable, and the security reduction is tight. (In particular, the security reduction does not lose a factor of $|\mathcal{I}|$, where $|\mathcal{I}|$ is the number of possible challenges sent by the verifier.)

We stress that, since the set of commit-choose-open protocols is closed under parallel composition, we get composability "for free." Now witness indistinguishable argument systems already enjoy a composition theorem (see, e.g., Goldreich [17, Lemma 4.6.6]), so the compositionality claim is not surprising. However, we believe that our results demonstrate that the security notion of IND-SO-COM secure commitments itself is a reasonable fallback to SIM-SO-COM security.

Auxiliary-input IND-SO-COM security. Since the standard definition of witness indistinguishability (see Definition 2.6 involves an auxiliary input $z$ given to the verifier/adversary $V^{*}$, we also consider a variation of Definition 4.1 that involves auxiliary input. Namely,

Definition 6.6 (AI-IND-SO-COM). In the situation of Definition 4.1, we say that Com is AIIND-SO-COM secure iff $\mathrm{Adv}_{\mathrm{Com}, \mathcal{M}, A, z}^{\text {ind-so }}$ is negligible for all PPT $\mathcal{M}$ and $A$ and all auxiliary inputs $z=\left(z_{k}\right)_{k \in \mathbb{N}} \in\left(\{0,1\}^{*}\right)^{\mathbb{N}}$, where both $\mathcal{M}$ and $A$ are invoked with additional auxiliary input $z_{k}$.

We stress that the proof of Theorem 4.11 shows AI-IND-SO-COM security, once the investigated commitment scheme is statistically hiding against non-uniform adversaries.

Now we are ready to prove the following connection between witness indistinguishability and AI-IND-SO-COM:

Theorem 6.7 (AI-IND-SO-COM implies witness indistinguishability). Assume a CCO protocol IP with parameters $n^{\prime}$ and $\mathcal{I}^{\prime}$ that uses commitment scheme Com as in Definition 6.1. If Com is AI-IND-SO-COM for parameters $n=n^{\prime}+1$ and $\mathcal{I}=\mathcal{I}^{\prime}$, then IP is witness indistinguishable. The security reduction loses only a factor of 2. 
Proof. Assume arbitrary $x, w^{0}, w^{1}, V^{*}, D, z$ as in Definition 2.6. We construct a message distribution $\mathcal{M}$, an adversary $A$, and a $z^{\prime}$ such that

$$
\operatorname{Adv}_{\mathrm{Com}, \mathcal{M}, A, z}^{\text {ind-so }}=\frac{1}{2} \operatorname{Adv}_{x, w^{0}, w^{1}, V^{*}, D, z}^{\mathrm{WI}} .
$$

First, define $z_{k}^{\prime}=\left(x_{k}, w_{k}^{0}, w_{k}^{1}, z_{k}\right)$, so that $\mathcal{M}$ and $A$ are both invoked with both witnesses and $z_{k}$. Then, let $\mathcal{M}$ be the following PPT algorithm:

1. upon input $z_{k}^{\prime}=\left(x_{k}, w_{k}^{0}, w_{k}^{1}, z_{k}\right)$, toss a coin $b \in\{0,1\}$,

2. sample messages $\left(M_{i}\right)_{i \in\left[n^{\prime}\right]}$ by running $\mathrm{P}$ on input $\left(x_{k}, w_{k}^{b}\right)$,

3. define $M_{n^{\prime}+1}:=b$,

4. return the $\left(n^{\prime}+1\right)$-message vector $\left(M_{i}\right)_{i \in\left[n^{\prime}+1\right]}$.

Now adversary $A$, running in the IND-SO-COM experiment, proceeds as follows:

1. upon input $z_{k}^{\prime}=\left(x_{k}, w_{k}^{0}, w_{k}^{1}, z_{k}\right)$, start an internal simulation of $V^{*}$ on input $\left(x_{k}, z_{k}\right)$,

2. upon receiving $n=n^{\prime}+1$ Com-commitments from the experiment, relay the first $n^{\prime}$ of these commitments to $V^{*}$, and receive the $\left(n^{\prime}+1\right)$-th commitment,

3. when $V^{*}$ chooses a set $I \subseteq\left[n^{\prime}\right]$, relay this set (interpreted as a subset of $[n]=\left[n^{\prime}+1\right]$ ) to the experiment,

4. upon receiving openings (for $i \in I$ ) from the experiment, relay these openings to $V^{*}$,

5. when the interaction between experiment and $V^{*}$ finishes, run $b^{\prime} \leftarrow D\left(x_{k}, z_{k}, T\right)$ to obtain a bit $b^{\prime}$, where $T$ denotes the transcript of the interaction between the experiment and $V^{*}$,

6. upon receiving a message vector $M^{*}=\left(M_{i}^{*}\right)_{i \in[n]}$ from the experiment, output $b^{\prime} \oplus M_{n^{\prime}+1}^{*}$.

Now in the real IND-SO-COM experiment $\operatorname{Exp}_{\operatorname{Com}, \mathcal{M}, A, z}^{\text {ind-roal }}$, the following happens: if $\mathcal{M}$ chose $b=0$, then an interaction of $\mathrm{P}\left(x_{k}, w_{k}^{0}\right)$ and $V^{*}\left(x_{k}, z_{k}\right)$ is perfectly simulated. Since $M_{n^{\prime}+1}^{*}=b=0$,

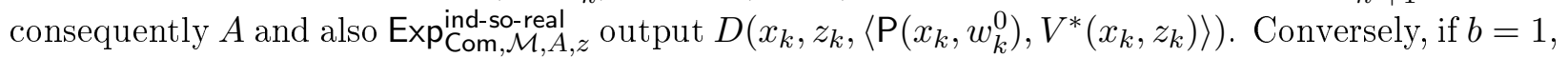

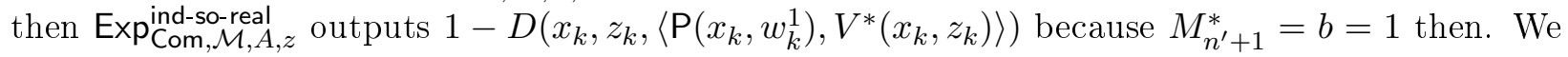
get that

$$
\begin{aligned}
\operatorname{Pr}\left[\operatorname{Exp}_{\text {Com }, \mathcal{M}, A, z}^{\text {ind-so-real }}=1\right] & =\frac{1}{2}\left(\operatorname{Pr}\left[D\left(x_{k}, z_{k},\left\langle\mathrm{P}\left(x_{k}, w_{k}^{0}\right), V^{*}\left(x_{k}, z_{k}\right)\right\rangle\right)=1\right]\right. \\
& \left.+1-\operatorname{Pr}\left[D\left(x_{k}, z_{k},\left\langle\mathrm{P}\left(x_{k}, w_{k}^{0}\right), V^{*}\left(x_{k}, z_{k}\right)\right\rangle\right)=1\right]\right)=\frac{1}{2} \mathrm{Adv}_{x, w^{0}, w^{1}, V^{*}, D, z}^{\mathrm{WI}}+\frac{1}{2} .
\end{aligned}
$$

On the other hand, in the ideal IND-SO-COM experiment, the message $M_{n^{\prime}+1}^{*}$ that $A$ receives from the experiment results from a resampling of $\mathcal{M}$, conditioned on $M_{I}^{*}=M_{I}$. Since IP is a CCO protocol, $M_{I}$ is independent of the used witness. Hence $M_{I}$ is also independent of $b$, and so $M_{n^{\prime}+1}^{*}$ will be a freshly tossed coin. We get

$$
\operatorname{Pr}\left[\operatorname{Exp}_{\mathrm{Com}, \mathcal{M}, A, z}^{\text {ind-so-ideal }}=1\right]=\frac{1}{2} .
$$

Putting things together proves the theorem.

Tightness in the reduction and composition. We stress that we only lose a factor of 2 in our security reduction, which contrasts the loss of a factor of about $n^{\prime 2}$ in the proof of Goldreich et al. 20. Their proof works also for perfectly binding commitment schemes (thus achieving an interactive proof system), which we (almost) cannot hope to satisfy AI-IND-SO-COM security, according to Theorem 4.2 However, since we can instantiate AI-IND-SO-COM secure schemes for arbitrary parameters $n$ and $\mathcal{I}$, we can hope to apply Theorem 6.7 even to protocols where 
$\left|\mathcal{I}_{n}\right|$ is super-polynomial ${ }^{11}$ In particular, we can apply our theorem to a parallel composition of a CCO protocol (which is again a CCO protocol). This gives a composition theorem for the witness indistinguishability of CCO protocols (implemented with AI-IND-SO-COM secure commitments) at virtually no extra cost.

\section{Conclusion and open problems}

While our results give an almost complete characterization when and how security under selective openings can be achieved, some interesting questions remain. Most importantly:

- Is there a non-interactive commitment scheme which is simulatable under selective openings? The existence of such a scheme would prove the existence of 3-round zero-knowledge proofs with negligible soundness error. While Theorem 3.3 states that any such scheme must employ nonstandard techniques, we cannot rule out such schemes completely.

Another interesting question is the following:

- Are statistically hiding commitment schemes simulatable under selective openings? Theorem 4.11 states that such schemes are at least indistinguishable under selective openings. However, our proof gives no indication on how to construct a simulator.

\section{Acknowledgements.}

I am indebted to Enav Weinreb, Marc Stevens, Serge Fehr, and Krzysztof Pietrzak for many insightful discussions. In particular, Enav suggested interactive proof systems as an application, and Marc contributed to the first negative result.

\section{References}

[1] Boaz Barak. How to go beyond the black-box simulation barrier. In 42th Annual Symposium on Foundations of Computer Science, Proceedings of FOCS 2001, pages 106-115. IEEE Computer Society, 2001.

[2] Boaz Barak and Oded Goldreich. Universal arguments and their applications. In 17th Annual IEEE Conference on Computational Complexity, Proceedings of CoCo 2002, pages 194-203. IEEE Computer Society, 2002.

[3] Boaz Barak, Manoj Prabhakaran, and Amit Sahai. Concurrent non-malleable zero-knowledge. In 47th Annual Symposium on Foundations of Computer Science, Proceedings of FOCS 2006, pages 345-354. IEEE Computer Society, 2006.

[4] Donald Beaver. Plug and play encryption. In Joan Feigenbaum, editor, Advances in Cryptology, Proceedings of CRYPTO '91, number 576 in Lecture Notes in Computer Science, pages 75-89. Springer-Verlag, 1992.

[5] Mihir Bellare and Phillip Rogaway. Optimal asymmetric encryption-how to encrypt with RSA. In Alfredo de Santis, editor, Advances in Cryptology, Proceedings of EUROCRYPT '94, number 950 in Lecture Notes in Computer Science, pages 92-111. Springer-Verlag, 1995.

\footnotetext{
${ }^{11}$ Of course, it is possibly to directly prove, say, witness indistinguishability for the case of super-polynomial $\left|\mathcal{I}_{n}\right|$ from statistically hiding commitment schemes. However, our point here is to illustrate the usefulness of our definition.
} 
[6] Manuel Blum. Coin flipping by telephone. In Allen Gersho, editor, Advances in Cryptology, A report on CRYPTO 81, number 82-04 in ECE Report, pages 11-15. University of California, Electrical and Computer Engineering, 1982.

[7] Ran Canetti, Uri Feige, Oded Goldreich, and Moni Naor. Adaptively secure multi-party computation. In Twenty-Eighth Annual ACM Symposium on Theory of Computing, Proceedings of STOC 1995, pages 639-648. ACM Press, 1996.

[8] Ran Canetti, Cynthia Dwork, Moni Naor, and Rafail Ostrovsky. Deniable encryption. In Burton S. Kaliski Jr., editor, Advances in Cryptology, Proceedings of CRYPTO '97, number 1294 in Lecture Notes in Computer Science, pages 90-104. Springer-Verlag, 1997.

[9] Ran Canetti, Joe Kilian, Erez Petrank, and Alon Rosen. Concurrent zero-knowledge requires $\tilde{\Omega}(\log n)$ rounds. In 33th Annual ACM Symposium on Theory of Computing, Proceedings of STOC 2001, pages 570-579. ACM Press, 2001.

[10] Ran Canetti, Shai Halevi, and Jonathan Katz. Adaptively-secure, non-interactive public-key encryption. In Joe Kilian, editor, Theory of Cryptography, Proceedings of TCC 2005, number 3378 in Lecture Notes in Computer Science, pages 150-168. Springer-Verlag, 2005.

[11] Ivan Damgård. A "proof-reading" of some issues in cryptography. In Lars Arge, Christian Cachin, Tomasz Jurdzinski, and Andrzej Tarlecki, editors, Automata, Languages and Programming, 34th International Colloquium, Proceedings of ICALP 200\%, number 4596 in Lecture Notes in Computer Science, pages 2-11. Springer-Verlag, 2007.

[12] Ivan Damgård and Jesper Buus Nielsen. Improved non-committing encryption schemes based on general complexity assumptions. In Mihir Bellare, editor, Advances in Cryptology, Proceedings of CRYPTO 2000, number 1880 in Lecture Notes in Computer Science, pages 432-450. Springer-Verlag, 2000.

[13] Ivan B. Damgård, Torben P. Pedersen, and Birgit Pfitzmann. On the existence of statistically hiding bit commitment schemes and fail-stop signatures. In Douglas R. Stinson, editor, Advances in Cryptology, Proceedings of CRYPTO '93, number 773 in Lecture Notes in Computer Science, pages 250-265. Springer-Verlag, 1994.

[14] Yevgeniy Dodis, Roberto Oliveira, and Krzysztof Pietrzak. On the generic insecurity of the full domain hash. In Victor Shoup, editor, Advances in Cryptology, Proceedings of CRYPTO 2005, number 3621 in Lecture Notes in Computer Science, pages 449-466. Springer-Verlag, 2005.

[15] Cynthia Dwork, Moni Naor, Omer Reingold, and Larry Stockmeyer. Magic functions. Journal of the ACM, 50(6):852-921, 2003.

[16] Cynthia Dwork, Moni Naor, and Amit Sahai. Concurrent zero-knowledge. Journal of the ACM, 51(6):851-898, 2004.

[17] Oded Goldreich. Foundations of Cryptography - Volume 1 (Basic Tools). Cambridge University Press, August 2001.

[18] Oded Goldreich and Ariel Kahan. How to construct constant-round zero-knowledge proof systems for np. Journal of Cryptology, 9(3):167-190, 1996. 
[19] Oded Goldreich and Hugo Krawczyk. On the composition of zero-knowledge proof systems. In Mike Paterson, editor, Automata, Languages and Programming, 17th International Colloquium, Proceedings of ICALP 90, number 443 in Lecture Notes in Computer Science, pages 268-282. Springer-Verlag, 1990.

[20] Oded Goldreich, Silvio Micali, and Avi Wigderson. Proofs that yield nothing but their validity or all languages in NP have zero-knowledge proof systems. Journal of the ACM, 38(1):691-729, 1991.

[21] Iftach Haitner and Omer Reingold. Statistically-hiding commitment from any one-way function. In 39th Annual ACM Symposium on Theory of Computing, Proceedings of STOC 2007, pages 1-10. ACM Press, 2007.

[22] Russell Impagliazzo and Steven Rudich. Limits on the provable consequences of one-way permutations. In Twenty-First Annual ACM Symposium on Theory of Computing, Proceedings of STOC 1989, pages 44-61. ACM Press, 1989. Extended abstract.

[23] Joe Kilian and Erez Petrank. Concurrent and resettable zero-knowledge in poly-logarithmic rounds. In 33th Annual ACM Symposium on Theory of Computing, Proceedings of STOC 2001, pages 560-569. ACM Press, 2001.

[24] Moni Naor. Bit commitment using pseudo-randomness. Journal of Cryptology, 4(2):151-158, 1991.

[25] Moni Naor and Moti Yung. Universal one-way hash functions and their cryptographic applications. In Twenty-First Annual ACM Symposium on Theory of Computing, Proceedings of STOC 1989, pages 33-43. ACM Press, 1989.

[26] Jesper B. Nielsen. Separating random oracle proofs from complexity theoretic proofs: The non-committing encryption case. In Moti Yung, editor, Advances in Cryptology, Proceedings of CRYPTO 2002, number 2442 in Lecture Notes in Computer Science, pages 111-126. SpringerVerlag, 2002.

[27] Omer Reingold, Luca Trevisan, and Salil P. Vadhan. Notions of reducibility between cryptographic primitives. In Moni Naor, editor, Theory of Cryptography, Proceedings of TCC 2004, number 2951 in Lecture Notes in Computer Science, pages 1-20. Springer-Verlag, 2004.

[28] Ransom Richardson and Joe Kilian. On the concurrent composition of zero-knowledge proofs. In Jacques Stern, editor, Advances in Cryptology, Proceedings of EUROCRYPT '99, number 1592 in Lecture Notes in Computer Science, pages 415-431. Springer-Verlag, 1999.

[29] Daniel R. Simon. Finding collisions on a one-way street: Can secure hash functions be based on general assumptions? In Kaisa Nyberg, editor, Advances in Cryptology, Proceedings of EUROCRYPT '98, number 1403 in Lecture Notes in Computer Science, pages 334-345. SpringerVerlag, 1998.

\section{A On the role of property $\mathcal{P}$}

The intuitive contradiction. The formulations of Theorem 3.3 and Theorem 4.2 seem intuitively much too general: essentially they claim impossibility of black-box proofs from any computational 
assumption which is formulated as a property $\mathcal{P}$ of an oracle $\mathcal{X}$. Why can't we choose $\mathcal{X}$ to be an ideally secure commitment scheme, and $\mathcal{P}$ a property that models precisely what we want to achieve, e.g., Definition 4.1 (i.e., IND-SO-COM security)? After all, Definition 4.1 can be rephrased as a property $\mathcal{P}$ by letting $A$ choose a message distribution $\mathcal{M}$ and send this distribution (as a description of a PPT algorithm $\mathcal{M}$ ) to $\mathcal{P}$. Then, $\mathcal{P}$ could perform the $\operatorname{Exp}_{\mathrm{Com}, \mathcal{M}, A}^{\text {ind-so-ral }}$ or the $\operatorname{Exp}_{\mathrm{Com}, \mathcal{M}, A}^{\text {ind-so-ideal }}$ experiment with $A$, depending on an internal coin toss (the output of $\mathcal{P}$ will then depend on $A$ 's output and on that coin toss). This $\mathcal{P}$ models Definition 4.1, in the sense that

$$
\operatorname{Adv}_{\mathrm{Com}, \mathcal{M}, A}^{\text {ind-so }}=2 \operatorname{Adv}_{\mathcal{P}, \mathcal{X}, A}^{\text {prop }}
$$

Also, using a truly random permutation as a basis, it is natural to assume that we can construct an ideal (i.e., as an oracle) perfectly binding commitment scheme $\mathcal{X}$ that satisfies $\mathcal{P}$. (Note that although $\mathcal{X}$ is perfectly binding, $A$ 's view may still be almost statistically independent of the unopened messages, since the scheme $\mathcal{X}$ is given in oracle form.)

Hence, if the assumption essentially is already IND-SO-COM security, we can certainly achieve IND-SO-COM security (using a trivial reduction), and this seems to contradict Theorem 4.2. So where is the problem?

Resolving the situation. The problem in the above argument is that $\mathcal{P}$-security (our assumption) implies IND-SO-COM security (our goal) in a fundamentally non-black-box way. Namely, the proof converts an IND-SO-COM adversary $A$ and a message distribution $\mathcal{M}$ into a $\mathcal{P}$-adversary $A^{\prime}$ that sends a description of $\mathcal{M}$ to $\mathcal{P}$. This very step makes use of an explicit representation of the message distribution $\mathcal{M}$, and this is what makes the whole proof non-black-box. In other words, this way of achieving IND-SO-COM security cannot be black-box, and there is no contradiction to our results.

Viewed from a different angle, the essence of our impossibility proofs is: build a very specific message distribution, based on oracles $(\mathcal{R O}$, resp. $\mathcal{C})$, such that another "breaking oracle" $\mathcal{B}$ "breaks" this message distribution if and only if the adversary can prove that he can open commitments. This step relies on the fact that we can specify message distributions which depend on oracles. Relative to such oracles, property $\mathcal{P}$ still holds (as we prove), but may not reflect IND-SO-COM security anymore. Namely, since $\mathcal{P}$ itself cannot access additional oracles $12, \mathcal{P}$ is also not able to sample a message space that depends on additional (i.e., on top of $\mathcal{X}$ ) oracles. So in our reduction, although $A$ itself can, both in the IND-SO-COM experiment and when interacting with $\mathcal{P}$, access all oracles, it will not be able to communicate a message distribution $\mathcal{M}$ that depends on additional oracles (on top of $\mathcal{X}$ ) to $\mathcal{P}$. On the other hand, any PPT algorithm $\mathcal{M}$, as formalized in Definition 4.1, can access all available oracles.

So for the above modeling of IND-SO-COM security as a property $\mathcal{P}$ in the sense of Definition 3.2, our impossibility results still hold, but become meaningless (since basically using property $\mathcal{P}$ makes the proof non-black-box). In a certain sense, this comes from the fact that the modeling of IND$\mathrm{SO}-\mathrm{COM}$ as a property $\mathcal{P}$ is inherently non-black-box.

What computational assumptions can be formalized as properties in a "black-box" way? Fortunately, most standard computational assumptions can be modeled in a black-box way as a property $\mathcal{P}$. Besides the mentioned one-way property (and its variants), in particular, e.g., the IND-CCA security game for encryption schemes can be modeled. Observe that in this game, we can let the IND-CCA adversary himself sample challenge messages $M_{0}, M_{1}$ for the IND-CCA

\footnotetext{
${ }^{12}$ by definition, $\mathcal{P}$ must be specified independently of additional oracles, cf. Definition 3.2 if we did allow $\mathcal{P}$ to access additional oracles, this would break our impossibility proofs
} 
experiment from his favorite distribution; no PPT algorithm has to be transported to the security game. In fact, the only properties which do not allow for black-box proofs are those that involve an explicit transmission of code (i.e., a description of a circuit or a Turing machine). In that sense, the formulation of Theorem 3.3 and Theorem 4.2 is very general and useful.

(Non-)programmable random oracles. We stress that the black-box requirement for random oracles (when used in the role of $\mathcal{X}$ ) corresponds to "non-programmable random oracles" (as used by, e.g., Bellare and Rogaway [5]) as opposed to "programmable random oracles" (as used by, e.g., Nielsen [26]). Roughly, a proof in the programmable random oracle model translates an attack on a cryptographic scheme into an attack on a simulated random oracle (that is, an oracle completely under control of simulator). Naturally, such a reduction is not black-box. And indeed, with programmable random oracles, even non-interactive SIM-SO-COM secure commitment schemes can be built relatively painless. As an example, [26] proves a simple encryption scheme (which can be interpreted as a non-interactive commitment scheme) secure under selective openings. 